\title{
Multi-Symplectic Magnetohydrodynamics
}

\author{
G. M. WEB B ${ }^{1} \dagger$, J.F. McKENZIE $\mathbf{E}^{1,3}$ \\ AND G. P. Z A N K $\mathbf{K}^{1,2}$ \\ ${ }^{1}$ Center for Space Plasma and Aeronomic Research, The University of Alabama in Huntsville, \\ Huntsville AL 35805, USA \\ ${ }^{2}$ Department of Space Science, The University of Alabama in Huntsville, Huntsville AL 35805, \\ USA \\ ${ }^{3}$ Department of Mathematics and Statistics, Durban University of Technology, \\ Steve Biko Campus, Durban South Africa, and School of Mathematical Sciences,University of \\ Kwa-Zulu Natal, Durban South Africa
}

(Received 24 April 2014)

A multi-symplectic formulation of ideal magnetohydrodynamics (MHD) is developed based on a Clebsch variable variational principle in which the Lagrangian consists of the kinetic minus the potential energy of the MHD fluid modified by constraints using Lagrange multipliers, that ensure mass conservation, entropy advection with the flow, the Lin constraint and Faraday's equation (i.e the magnetic flux is Lie dragged with the flow). The analysis is also carried out using the magnetic vector potential $\tilde{\mathbf{A}}$ where $\alpha=\tilde{\mathbf{A}} \cdot d \mathbf{x}$ is Lie dragged with the flow, and $\mathbf{B}=\nabla \times \tilde{\mathbf{A}}$. The multi-symplectic conservation laws give rise to the Eulerian momentum and energy conservation laws. The symplecticity or structural conservation laws for the multi-symplectic system corresponds to the conservation of phase space. It corresponds to taking derivatives of the momentum and energy conservation laws and combining them to produce $n(n-1) / 2$ extra conservation laws, where $n$ is the number of independent variables. Noether's theorem for the multi-symplectic MHD system is derived, including the case of non-Cartesian space coordinates, where the metric plays a role in the equations.

\section{Introduction}

Multi-symplectic equations for Hamiltonian systems with two or more independent variables $x^{\alpha}$ have been developed as a useful extension of Hamiltonian systems with one evolution variable $t$. This development has connections with dual variational formulations of travelling wave problems (e.g. Bridges (1992)), and is useful in numerical schemes for Hamiltonian systems. Bridges and co-workers used the multi-symplectic approach to study linear and nonlinear wave propagation, generalizations of wave action, wave modulation theory, and wave stability problems (Bridges (1997a,b)). Reich (2000) and Bridges (2006) develop multi-symplectic difference schemes. Multi-symplectic Hamiltonian systems have been studied by Marsden and Shkoller (1999) and Bridges et al. (2005). Webb et al. (2007); Webb et al. (2008, 2014d) discuss travelling waves in multi-fluid plasmas using a multi-symplectic formulation analogous to Bridges (1992) paper on travelling water waves. Holm et al. (1998) give an overview of Hamiltonian systems, semi-direct product Lie algebras and Euler-Poincaré equations.

Cotter et al. (2007) developed a multi-symplectic, Euler-Poincaré formulation of fluid mechanics. They showed that multi-symplectic ideal fluid mechanics type systems are related to Clebsch variable formulations in which the Lagrange multipliers play the role

$\dagger$ Email address for correspondence: gmw0002@uah.edu 
of canonically conjugate momenta to the constrained variables (Zakharov and Kuznetsov (1997), Morrison (1998)). Thus, the Clebsch variable formulation involves a momentum map. As a part of the paper, we give a brief introduction to multi-symplectic systems (Sections 3 and 4), based on the work of Hydon (2005) and Cotter et al. (2007) (see also Brio et al. (2010)). In multi-symplectic Hamiltonian systems, both the space and the time variables can be thought of as evolution variables. In this paper we obtain multisymplectic equations for ideal gas dynamics and MHD, based on the Clebsch variables formulation. The energy and momentum conservation laws for gas dynamics and MHD are obtained from the multi-symplectic formalism. We also discuss and give examples of the symplecticity or structural conservation laws, which are obtained by combining derivatives of the energy and momentum conservation laws. The symplecticity conservation law thus impose higher order derivative constraints on the energy and momentum conservation laws.

The present paper is related to recent work by Webb et al. (2014a); Webb et al. (2014b); Webb et al. (2014c) and Webb and Mace (2014) on conservation laws, Hamiltonian and symmetry group approaches to the MHD equations. In Webb et al. (2014a) advected invariants in ideal fluid mechanics and MHD were obtained by using Lie dragging techniques (see also Tur and Yanovsky (1993)). In particular helicity conservation laws were discussed (i.e. fluid helicity conservation in ideal fluids (e.g. Moffatt (1969)), cross helicity and magnetic helicity in MHD (Woltjer (1958)). Berger and Field (1984) investigated magnetic helicity and relative magnetic helicity (see also Finn and Antonsen (1985, 1988)). Berger (1990), Ruzmaikin and Akhmetiev (1994) and Akhmetiev and Ruzmaikin (1995) investigated higher order MHD knot invariants known as Sato-Levine invariants, which can be described using Seifert surfaces (e.g. used to describe the Whitehead link and Borromean rings). For special ideal MHD flows in which the magnetic helicity density $\mathbf{A} \cdot \mathbf{B}$ vanishes, there is a higher order magnetic topological charge, namely the Godbillon Vey invariant (e.g. Tur and Yanovsky (1993), Webb et al. (2014a); Webb et al. (2014c)) which also describes the magnetic field topology (i.e. magnetic helicity is not the only quantity describing magnetic topology). For the Godbillon Vey invariant, the gauge of the magnetic vector potential $\mathbf{A}$ is chosen so that the one-form $\alpha=\mathbf{A} \cdot d \mathbf{x}$ is Lie dragged by the background plasma flow. Webb et al. (2014b) derive MHD conservation laws using Noether's first and second theorems (see Hydon and Mansfield (2011) for an insightful treatment of Noether's second theorem). In the most general case, the fluid helicity and cross helicity conservation laws are nonlocal as they involve Clebsch potentials, which are nonlocal variables (e.g. Webb et al. (2014a); Webb et al. (2014b)). A brief synopsis of this work is given by Webb et al. (2014c). Webb and Mace (2014) use Noether's second theorem and fluid relabelling symmetries to derive conservation laws in MHD using the approach of Hydon and Mansfield (2011). They obtain a new potential vorticity type conservation law for MHD using a non-field aligned fluid relabelling symmetry of the equations.

The main aim of the present paper is to provide a multi-symplectic formulation of the MHD equations by using Clebsch variables.

In Section 2 the basic ideal MHD equations are given. In Section 2, we give a simple example of the multi-symplectic form of the ideal gas dynamic equations in one Cartesian space coordinate. We give a recipe for constructing the multi-symplectic form of the equations and describe the symplecticity or structural conservation law for 1D gas dynamics. By using Clebsch variables, one is in effect increasing the number of dependent variables describing the system. To ensure that the system of equations describes the original Eulerian fluid equations, extra constraints or conservation laws (the symplecticity 
conservation laws) ensures that original system of equations is obtained. It turns out that the symplecticity conservation equations can be expressed in terms of two-dimensional Jacobians of the dependent variables and the independent space and time variables. The basic theory behind this approach is described in Section 4.

In Section 3, we give an introduction to Clebsch variables and momentum maps following the analysis of Cotter et al. (2007). We introduce the Clebsch variable description of MHD (e.g. Zakharov and Kuznetsov (1997), Morrison (1998), Holm and Kupershmidt (1983a,b), Webb et al. (2014a); Webb et al. (2014b)). We give two different formulations of the MHD variational principle. In the first formulation, Faraday's law for the magnetic field induction $\mathbf{B}$ is included as a constraint. The constraints of mass continuity, entropy advection, and the Lin constraint are incorporated in the variational principle by means of Lagrange multipliers. In a second variational formulation, the Faraday equation constraint is replaced by the condition that the magnetic vector potential 1-form: $\alpha=\tilde{\mathbf{A}} \cdot d \mathbf{x}$ is Lie dragged by the flow, where $\mathbf{B}=\nabla \times \tilde{\mathbf{A}}$ (e.g. Tur and Yanovsky (1993), Gordin and Petviashvili (1987), Padhye and Morrison (1996a,b) and Webb et al. (2014a)). In the case $\nabla \cdot \mathbf{B}=0$, and $\mathbf{B}=\nabla \times \tilde{\mathbf{A}}$, the condition that the one-form $\alpha=\tilde{\mathbf{A}} \cdot d \mathbf{x}$ is Lie dragged by the flow is equivalent to Faraday's equation. We include an extra $\mathbf{u} \nabla \cdot \mathbf{B}$ term in Faraday's equation for mathematical reasons. This allows one to obtain a mapping between the multi-symplectic MHD equations using the advected A formalism and the advected magnetic flux version of Faraday's law (see Proposition (5.3) and (6.7) in the conclusions). It is also useful to include the $\mathbf{u} \nabla \cdot \mathrm{B}$ in numerical MHD in order to minimize numerically generated $\nabla \cdot \mathrm{B}$ in numerical MHD codes by advecting the numerically generated $\nabla \cdot \mathbf{B} \neq 0$ out of the computational domain (e.g. Powell et al. (1999), Webb et al. (2010)). By setting $\nabla \cdot \mathbf{B}=0$ after all calculations are done gives rise to correct physical results, but allows one to retain a mathematically more useful form of the equations. In general, Faraday's equation is equivalent to the condition that the magnetic field flux 2 -form $\beta=\mathbf{B} \cdot d \mathbf{S}$ is Lie dragged with the flow. If $\nabla \cdot \mathbf{B}=0$ then $\beta=d \alpha$ where $d \alpha$ is the exterior derivative of the 1 -form $\alpha$.

Section 4 presents an overview of multi-symplectic Hamiltonian systems based in part on the work of Hydon (2005). We discuss the symplecticity conservation laws, which are related to the conservation of phase space in multi-symplectic systems, in which the generalized phase space volume element $\kappa^{\alpha}=(1 / 2) \mathrm{K}_{i j}^{\alpha} d z^{i} \wedge d z^{j}$ is given by the exterior derivative of a one-form $\omega^{\alpha}$. This requirement implies $d \kappa^{\alpha}=d d \omega^{\alpha}=0$ (by the Poincaré Lemma), which implies the conservation of the phase space element $\kappa^{\alpha}$ where $z^{i}$ are the canonical variables. We also discuss Noether's first theorem for multi-symplectic systems. We describe the form of multi-symplectic systems for the case of non-Cartesian spatial coordinates where the spatial metric plays a role in the equations (see also Bridges et al. (2010)).

Section 5 develops the multi-symplectic MHD evolution equations. The analysis is similar to that of Cotter et al. (2007) where they develop the multi-symplectic approach to Hamiltonian hydrodynamic type systems.

Section 6 concludes with a summary and discussion. 


\section{The Model}

The magnetohydrodynamic equations can be written in the form:

$$
\begin{aligned}
& \frac{\partial \rho}{\partial t}+\nabla \cdot(\rho \mathbf{u})=0, \\
& \frac{\partial}{\partial t}(\rho \mathbf{u})+\nabla \cdot\left[\rho \mathbf{u u}+\left(p+\frac{B^{2}}{2 \mu}\right) \mathbf{I}-\frac{\mathbf{B B}}{\mu}\right]=0, \\
& \frac{\partial S}{\partial t}+\mathbf{u} \cdot \nabla S=0, \\
& \frac{\partial \mathbf{B}}{\partial t}-\nabla \times(\mathbf{u} \times \mathbf{B})+\mathbf{u} \nabla \cdot \mathbf{B}=0,
\end{aligned}
$$

where $\rho, \mathbf{u}, p, S$ and $\mathbf{B}$ correspond to the gas density, fluid velocity, pressure, specific entropy, and magnetic induction $\mathbf{B}$ respectively, and $\mathbf{I}$ is the unit $3 \times 3$ dyadic. The gas pressure $p=p(\rho, S)$ is a function of the density $\rho$ and entropy $S$, and $\mu$ is the magnetic permeability. Equations (2.1)-(2.2) are the mass and momentum conservation laws, (2.3) is the entropy advection equation and (2.4) is Faraday's equation in the MHD limit.

In classical MHD, (2.1)-(2.4) are supplemented by Gauss' law:

$$
\nabla \cdot \mathbf{B}=0
$$

which implies the non-existence of magnetic monopoles.

It is useful to keep in mind the first law of thermodynamics:

$$
T d S=d Q=d U+p d V \quad \text { where } \quad V=\frac{1}{\rho},
$$

where $U$ is the internal energy per unit mass and $V=1 / \rho$ is the specific volume. Using the internal energy per unit volume $\varepsilon=\rho U$ instead of $U,(2.6)$ may be written as:

$$
T d S=\frac{1}{\rho}(d \varepsilon-h d \rho) \quad \text { where } \quad h=\frac{\varepsilon+p}{\rho},
$$

is the enthalpy of the gas. Assuming $\varepsilon=\varepsilon(\rho, S),(2.7)$ gives the formulae:

$$
\rho T=\varepsilon_{S}, \quad h=\varepsilon_{\rho}, \quad p=\rho \varepsilon_{\rho}-\varepsilon,
$$

relating the temperature $T$, enthalpy $h$ and pressure $p$ to the internal energy density $\varepsilon(\rho, S)$. From (2.7) we obtain:

$$
T d S=d h-\frac{1}{\rho} d p \quad \text { and } \quad-\frac{1}{\rho} \nabla p=T \nabla S-\nabla h,
$$

which is useful in the further analysis of the momentum equation for the system.

\subsection{Multi-Symplectic Example}

Multi-symplectic systems are a generalization of Hamiltonian systems. Consider the equations of $1 \mathrm{D}$ gas dynamics involving the variables $\mathbf{z}=(u, \rho, S, \beta, \phi)^{T}$ where

$$
u=\frac{\partial \phi}{\partial x}-\frac{\beta}{\rho} \frac{\partial S}{\partial x}
$$

is the Clebsch representation for the fluid velocity $u \equiv u^{x}$ directed along the $x$-axis. The governing equations have the form (e.g. Zakharov and Kuznetsov 
(1997), Morrison (1998)):

$$
\begin{aligned}
& \rho_{t}+(\rho u)_{x}=0, \quad S_{t}+u S_{x}=0, \\
& \phi_{t}+u \phi_{x}=\frac{1}{2} u^{2}-h, \quad \beta_{t}+(\beta u)_{x}=-\rho T,
\end{aligned}
$$

where $\varepsilon(\rho, S)$ is the the internal energy per unit volume of the gas. Here $\rho$, $T, p, h, S$ denote the density, temperature, pressure, enthalpy and entropy of the gas, where $h=\varepsilon_{\rho}, \rho T=\varepsilon_{S}$ and $p=\rho \varepsilon_{\rho}-\varepsilon$. The time evolution of a functional $F$ of the physical variables satisfies Hamilton's equations:

$$
F_{t}=\{F, H\}=\int d x\left(\frac{\delta F}{\delta \rho} \frac{\delta H}{\delta \phi}-\frac{\delta F}{\delta \phi} \frac{\delta H}{\delta \rho}+\frac{\delta F}{\delta S} \frac{\delta H}{\delta \beta}-\frac{\delta F}{\delta \beta} \frac{\delta H}{\delta S}\right),
$$

where $\{F, H\}$ is the canonical Poisson bracket and

$$
H=\int d x\left(\frac{1}{2} \rho u^{2}+\varepsilon(\rho, S)\right)
$$

is the Hamiltonian functional in which $u$ is given by (2.10).

Equations (2.10)-(2.12) can be written in the multi-symplectic form:

$$
\left(\mathrm{K}^{0} \frac{\partial}{\partial t}+\mathrm{K}^{1} \frac{\partial}{\partial x}\right) \mathbf{z}=\frac{\delta M}{\delta \mathbf{z}}
$$

where

$$
M=-\int d x \ell=-\int d x\left(\frac{1}{2} \rho u^{2}-\varepsilon(\rho, S)\right),
$$

is the multi-symplectic Hamiltonian (note $\ell$ is the Lagrange density of the fluid). The skew symmetric matrices $\mathrm{K}^{0}$ and $\mathrm{K}^{1}$ are given by:

$$
\mathrm{K}^{0}=\left(\begin{array}{ccccc}
0 & 0 & 0 & 0 & 0 \\
0 & 0 & 0 & 0 & -1 \\
0 & 0 & 0 & -1 & 0 \\
0 & 0 & 1 & 0 & 0 \\
0 & 1 & 0 & 0 & 0
\end{array}\right), \quad \mathrm{K}^{1}=\left(\begin{array}{ccccc}
0 & 0 & \beta & 0 & -\rho \\
0 & 0 & 0 & 0 & -u \\
-\beta & 0 & 0 & -u & 0 \\
0 & 0 & u & 0 & 0 \\
\rho & u & 0 & 0 & 0
\end{array}\right) \text {. }
$$

In the (2.15) there are two skew symmetric matrices $\mathrm{K}^{0}$ and $\mathrm{K}^{1}$, which shows that both space and time can be thought of as evolution variables. Bridges (1992, 1997a,b, 2006); Bridges et al. (2010) has championed the use of multisymplectic methods for nonlinear wave problems, both in numerical methods for solving nonlinear wave equations, wave action and wave stability problems, and Hamiltonian bifurcation theory (i.e wave stability theory depending on a bifurcation parameter). Cotter et al. (2007) develop multi-symplectic approaches to incompressible fluid dynamics, and other systems.

Below we illustrate the recipe for obtaining the multi-symplectic form (2.15)-(2.17) based on the results of Section 4.

The constrained Lagrangian associated with the Clebsch representation in the present example has the form:

$$
L=\frac{1}{2} \rho u^{2}-\varepsilon(\rho, S)+L_{z^{s}}^{\alpha} \frac{\partial z^{s}}{\partial x^{\alpha}},
$$

where the term:

$$
L_{z^{s}}^{\alpha} \frac{\partial z^{s}}{\partial x^{\alpha}}=\phi\left[\frac{\partial \rho}{\partial t}+\frac{\partial}{\partial x}(\rho u)\right]+\beta\left(\frac{\partial S}{\partial t}+u \frac{\partial S}{\partial x}\right),
$$


contains the Lagrangian constraints associated with the mass continuity equation and the entropy advection equation, and $\mathrm{z}=(u, \rho, S, \beta, \phi)^{T}$. In (2.19) we identify

$$
\begin{array}{ll}
L_{\rho}^{0}=\phi, & L_{\rho}^{1}=\phi u, \quad L_{u}^{1}=\phi \rho, \\
L_{S}^{0}=\beta, & L_{S}^{1}=\beta u .
\end{array}
$$

The one-forms:

$$
\omega^{\alpha}=L_{z^{s}}^{\alpha} d z^{s}, \quad(\alpha=0,1)
$$

using (2.20) are given by:

$$
\omega^{0}=\phi d \rho+\beta d S, \quad \omega^{1}=u(\phi d \rho+\beta d S)+\phi \rho d u .
$$

The exterior derivatives of the 1-forms (2.22) are:

$$
\begin{aligned}
& d \omega^{0}=d \phi \wedge d \rho+d \beta \wedge d S \equiv \frac{1}{2} \mathrm{~K}_{\alpha \beta}^{0} d z^{\alpha} \wedge d z^{\beta}, \\
& d \omega^{1}=d u \wedge(\beta d S-\rho d \phi)+u(d \phi \wedge d \rho+d \beta \wedge d S) \equiv \frac{1}{2} \mathrm{~K}_{\alpha \beta}^{1} d z^{\alpha} \wedge d z^{\beta} .
\end{aligned}
$$

The multi-symplectic matrices $\mathrm{K}_{\alpha \beta}^{0}$ and $\mathrm{K}_{\alpha \beta}^{1}$ given in (2.17) can be determined from (2.23).

The multi-symplectic formalism can be used to obtain conservation laws, by using the properties of the differential forms $\omega^{\alpha}$ defining the system (see Section 4). Conservation laws can also be obtained by using the multisymplectic version of Noether's theorem. In Appendix A, we show how the multi-symplectic approach gives rise to the energy and momentum conservation equations of $1 \mathrm{D}$ gas dynamics, namely:

$$
\begin{aligned}
& G_{0}=\frac{\partial}{\partial t}\left[\frac{1}{2} \rho u^{2}+\varepsilon(\rho, S)\right]+\frac{\partial}{\partial x}\left[\rho u\left(\frac{1}{2} u^{2}+h\right)\right]=0, \\
& G_{1}=-\left[\frac{\partial}{\partial t}\left(\rho u^{2}\right)+\frac{\partial}{\partial x}\left(p+\rho u^{2}\right)\right]=0 .
\end{aligned}
$$

The multi-symplectic approach also gives rise to the symplecticity or structural conservation laws. For the case of $1 \mathrm{D}$ gas dynamics, there is only one structural conservation law, namely:

$$
\frac{\partial D}{\partial t}+\frac{\partial F}{\partial x}=0
$$

where

$$
\begin{aligned}
D & =\frac{\partial(\phi, \rho)}{\partial(t, x)}+\frac{\partial(\beta, S)}{\partial(t, x)}, \\
F & =\rho \frac{\partial(\phi, u)}{\partial(t, x)}+u \frac{\partial(\phi, \rho)}{\partial(t, x)}+\beta \frac{\partial(u, S)}{\partial(t, x)}+u \frac{\partial(\beta, S)}{\partial(t, x)} \\
& \equiv \frac{\partial(u \phi, \rho)}{\partial(t, x)}+\frac{\partial(\rho \phi, u)}{\partial(t, x)}+\frac{\partial(u \beta, S)}{\partial(t, x)},
\end{aligned}
$$

where $\partial(\phi, \psi) / \partial(t, x)=\phi_{t} \psi_{x}-\phi_{x} \psi_{t}$ is the Jacobian of $\phi$ and $\psi$ with respect to $t$ and $x$. The symplecticity conservation law (2.26) corresponds to the conservation law:

$$
D_{x} G_{0}-D_{t} G_{1}=0
$$


where $D_{x} \equiv \partial / \partial x, D_{t} \equiv \partial / \partial t$ and $G_{0}=0$ and $G_{1}=0$ are the energy and momentum conservation equations $(2.24)$ and (2.25) written in terms of the Clebsch potentials.

The main point here, is that the multi-symplectic structure is determined by the fundamental one-forms $\omega^{\alpha}(\alpha=0,1)$ and the Hamiltonian functional $M$. The theory for this is outlined in Sections 3 and 4, and is used to determine the multi-symplectic structure of the MHD equations in Section 5. We give the generalization of the symplecticity conservation law in the general case for MHD and gas dynamics in Section 5.

\section{Hamiltonian Approach and Clebsch Variables}

In this section we first give a synopsis of the Clebsch variational principle and the inverse map, discussed in more detail in Cotter et al. (2007), who show how Clebsch type variational principles give rise to the momentum map (Section 3.1).

In Section 3.2 we describe a constrained variational principle for MHD using Lagrange multipliers to enforce the constraints of mass conservation; the entropy advection equation; Faraday's equation and the so-called Lin constraint describing in part, the vorticity of the flow (i.e. Kelvin's theorem). This leads to Hamilton's canonical equations in terms of Clebsch potentials (Zakharov and Kuznetsov (1997), Morrison (1998)). Morrison and Greene $(\mathbf{1 9 8 0}, \mathbf{1 9 8 2})$, and Morrison (1982) used the Clebsch variable formulation of MHD to derive the non-canonical Poisson bracket for MHD, by transforming the variational derivatives with respect to the Clebsch variables to their corresponding form in terms of Eulerian physical variables. Taking the variational derivative of the action with respect to the fluid velocity $u$, the Clebsch variational principle gives a representation for the fluid velocity $u$ in terms of the Clebsch potentials.

In Section 3.3 we transform the canonical Poisson bracket obtained from the Clebsch variable approach to a non-canonical Poisson bracket written in terms of Eulerian physical variables (see e.g. Morrison and Greene (1980, 1982), Morrison (1982), and Holm and Kupershmidt $(1983 \mathrm{a}, \mathrm{b}))$. We obtain the non-canonical Poisson brackets for MHD using the variables $(\mathbf{M}, \mathbf{B}, \rho, \sigma)$ where $\mathbf{M}=\rho \mathbf{u}$ is the MHD momentum flux, $\sigma=\rho S$ and $\mathbf{B}$ is the magnetic induction. We also use the non-canonical variables $(\mathbf{M}, \mathbf{A}, \rho, \sigma)$ where $\mathbf{A}$ is the magnetic vector potential in which the gauge is chosen so that the 1 -form $\alpha=\mathbf{A} \cdot d \mathbf{x}$ is an invariant advected with the flow.

\subsection{Clebsch Variables and the Momentum Map}

The Clebsch variational principle using the inverse map (i.e. Lagrangian map) involves the variational principle $\delta \mathcal{A}=0$ where

$$
\mathcal{A}=\int \ell[\mathbf{u}] d^{3} x d t+\int \boldsymbol{\pi} \cdot\left(\mathbf{l}_{t}+\mathbf{u} \cdot \nabla \mathbf{l}\right) d^{3} x d t .
$$

In (3.1) $\boldsymbol{\pi}$ is a Lagrange multiplier, ensuring that the Lagrange label $\mathbf{l}$ is advected with the fluid. In the present section we consider only the generic form of the Clebsch variational principle. More specific versions of the variational principle for MHD are discussed in later sections. 
The stationary point conditions for the variational functional (3.1) are:

$$
\begin{aligned}
& \frac{\delta \mathcal{A}}{\delta \mathbf{u}}=\frac{\delta \ell}{\delta \mathbf{u}}+(\nabla \mathbf{l})^{T} \cdot \boldsymbol{\pi}=0, \\
& \frac{\delta \mathcal{A}}{\delta \boldsymbol{\pi}}=\mathbf{l}_{t}+\mathbf{u} \cdot \nabla \mathbf{l}=0, \\
& \frac{\delta \mathcal{A}}{\delta \mathbf{l}}=-\left[\frac{\partial \boldsymbol{\pi}}{\partial t}+\nabla \cdot(\mathbf{u} \boldsymbol{\pi})\right]=0 .
\end{aligned}
$$

In ideal MHD

$$
\ell=\frac{1}{2} \rho|\mathbf{u}|^{2}-\varepsilon(\rho, S)-\frac{B^{2}}{2 \mu_{0}} \quad \text { and } \quad \frac{\delta \ell}{\delta \mathbf{u}}=\rho \mathbf{u} \equiv \mathbf{m},
$$

where $\mathbf{m}$ is the fluid momentum density or mass flux (see Section 3.2 for more details).

\subsubsection{Clebsch Momentum Map}

The momentum map $\mathbf{J}: T^{*} Q \rightarrow \mathfrak{g}^{*}$ from the cotangent bundle $T^{*} Q$ of the configuration manifold $Q$ to the dual $\mathfrak{g}^{*}$ of the Lie algebra $\mathfrak{g}$ of Lie group $G$ that acts on $Q$ defines a momentum map by the formula:

$$
\mathbf{J}\left(\nu_{q}\right) \cdot \boldsymbol{\xi}=\left\langle\nu_{q}, \boldsymbol{\xi}_{Q}(q)\right\rangle
$$

where $\nu_{q} \in T^{*} Q$ and $\boldsymbol{\xi} \in \mathfrak{g}$. Here $\boldsymbol{\xi}_{Q}$ is the infinitesimal generator of the Lie algebra element $\boldsymbol{\xi}$ action of $\boldsymbol{\xi}$ on $Q$ and $\left\langle\nu_{q}, \boldsymbol{\xi}_{Q}(q)\right\rangle$ is the pairing of an element of $T^{*} Q$ with an element of $T Q$.

For the case (3.1)-(3.4) the elements of $Q$ are the fluid labels $\mathbf{l}$ and the elements of $T^{*} Q$ are the conjugate pairs $(\mathbf{l}, \boldsymbol{\pi})$ of labels $\mathbf{l}$ and their conjugate momenta $\boldsymbol{\pi}$.

Proposition 3.1. The Clebsch relation (3.2) defines a right action $\operatorname{Diff}(\Omega)$ of diffeomorphisms on the domain $\Omega$ on the back to labels map $\mathbf{l}$.

Proof.Equation (3.2) defines a map $J_{\Omega}: T^{*} Q \rightarrow \mathfrak{X}^{*}(\Omega)$ from the cotangent bundle $T^{*} Q$ to the dual $\mathfrak{X}^{*}(\Omega)$ of vector fields on $\Omega$ :

$$
J_{\Omega}: \quad \mathbf{m} \cdot d \mathbf{x}=-\left((\nabla \mathbf{l})^{T} \cdot \boldsymbol{\pi}\right) \cdot d \mathbf{x}=-\boldsymbol{\pi} \cdot d \mathbf{l} \quad \text { where } \quad \mathbf{m}=\frac{\delta \ell}{\delta \mathbf{u}} .
$$

Thus, $\mathbf{J}_{\Omega}$ maps the conjugate pairs $(\mathbf{l}, \boldsymbol{\pi})$ to the space of 1 -form densities $\mathbf{m} \in \mathfrak{X}^{*}(\Omega)$. The map $J_{\Omega}$ may be associated with the smooth, invertible maps or diffeomorphisms $\boldsymbol{\eta}$ of the back-to-labels maps $\mathbf{l}$ by composition of functions $\operatorname{Diff}(\Omega): \mathbf{l} \cdot \boldsymbol{\eta}=\mathbf{l} \circ \boldsymbol{\eta}$. The effect of the map on the infinitesimal generators $X_{\Omega}(\mathbf{l})$ is formally defined as:

$$
X_{\Omega}(\mathbf{l}):=\left.\frac{d}{d s}(\mathbf{l} \circ \boldsymbol{\eta})(s)\right|_{s=0}=T \mathbf{l} \circ X
$$

where the differentiation with respect to $s$ is about the identity transformation at $s=0$. Equation (3.8) in component form is:

$$
\begin{aligned}
X_{\Omega}(\mathbf{l})^{i} \partial_{l^{i}} & =\frac{d}{d s} \mathbf{l}[\boldsymbol{\eta}(s)]^{i} \partial_{l^{i}}=\frac{\partial l^{i}}{\partial \eta^{k}} \frac{d \eta^{k}}{d s} \partial_{l^{i}}=\frac{d \eta^{k}}{d s}\left(\frac{\partial l^{i}}{\partial \eta^{k}} \frac{\partial}{\partial l^{i}}\right)=\frac{d \eta^{k}}{d s} \frac{\partial}{\partial \eta^{k}} \\
& =\frac{d \eta^{k}}{d s} \frac{\partial x^{\mu}}{\partial \eta^{k}} \frac{\partial}{\partial x^{\mu}}=\frac{d x^{\mu}}{d s} \frac{\partial}{\partial x^{\mu}} \equiv(T \mathbf{l} \circ X)^{i} \partial_{x^{i}} .
\end{aligned}
$$

From (3.9) it follows by the chain rule for differentiation that:

$$
X=\frac{d x^{\mu}}{d s} \frac{\partial}{\partial x^{\mu}}=\frac{d l^{i}}{d s} \frac{\partial}{\partial l^{i}}=\frac{d \eta^{k}}{d s} \frac{\partial}{\partial \eta^{k}}
$$


are equivalent forms for $X$.

The pairing between the map $\mathbf{J}_{\Omega}$ and the vector field $X \in \mathfrak{X}(\Omega)$ gives:

$$
\begin{aligned}
\left\langle\mathbf{J}_{\Omega}(\mathbf{l}, \boldsymbol{\pi}), X\right\rangle & =-\langle\boldsymbol{\pi} \cdot d \mathbf{l}, X\rangle=-\left\langle\pi_{k} d l^{k}, X^{j} \partial_{x^{j}}\right\rangle=-\left\langle\pi_{k} l_{, s}^{k} d x^{s}, X^{j} \partial_{x^{j}}\right\rangle \\
& =-\int \pi_{k} l_{, s}^{k} X^{s} d^{3} x=-\int \pi_{k}\left(X^{s} l_{, s}^{k}\right) d^{3} x \\
& =-\int \pi_{k} X\left(l^{k}\right) d^{3} x=-\int \pi_{k}(T \mathbf{l} \circ X)^{k} d^{3} x \\
& =-\int(\mathbf{l}, \boldsymbol{\pi})_{k}(T \mathbf{l} \circ X)^{k} d^{3} x=-\left\langle(\mathbf{l}, \boldsymbol{\pi}), X_{\Omega}(\mathbf{l})\right\rangle,
\end{aligned}
$$

Thus,

$$
\left\langle\mathbf{J}_{\Omega}(\mathbf{l}, \boldsymbol{\pi}), X\right\rangle=-\left\langle(\mathbf{l}, \boldsymbol{\pi}), X_{\Omega}(\mathbf{l})\right\rangle,
$$

which is equivalent to the defining relation (3.6) (the sign in (3.6) can be negative in the definition) for a momentum map.

\subsection{Clebsch variables and Hamilton's Equations}

Consider the MHD action (modified by constraints):

$$
J=\int d^{3} x d t L,
$$

where

$$
\begin{aligned}
L= & \left\{\frac{1}{2} \rho u^{2}-\epsilon(\rho, S)-\frac{B^{2}}{2 \mu_{0}}\right\}+\phi\left(\frac{\partial \rho}{\partial t}+\nabla \cdot(\rho \mathbf{u})\right) \\
& +\beta\left(\frac{\partial S}{\partial t}+\mathbf{u} \cdot \nabla S\right)+\lambda\left(\frac{\partial \mu}{\partial t}+\mathbf{u} \cdot \nabla \mu\right) \\
& +\boldsymbol{\Gamma} \cdot\left(\frac{\partial \mathbf{B}}{\partial t}-\nabla \times(\mathbf{u} \times \mathbf{B})+\mathbf{u}(\nabla \cdot \mathbf{B})\right) .
\end{aligned}
$$

The Lagrangian in curly brackets equals the kinetic minus the potential energy (internal thermodynamic energy plus magnetic energy). The Lagrange multipliers $\phi, \beta, \lambda$, and $\boldsymbol{\Gamma}$ ensure that the mass, entropy, Lin constraint, Faraday equations are satisfied. We do not enforce $\nabla \cdot \mathbf{B}=0$, since we are interested in the effect of $\nabla \cdot \mathbf{B} \neq 0$ (which is useful for numerical MHD where $\nabla \cdot \mathbf{B} \neq 0$ ). It is straightforward to impose $\nabla \cdot \mathbf{B} \neq 0$ if desired, and non-canonical Poisson brackets exist for this case as well (see (3.30) in this paper, and Morrison and Greene (1982)). Noncanonical Poisson brackets exist for this case as well as for the case $\nabla \cdot \mathbf{B}=0$ (e.g. Morrison and Greene (1982), Chandre et al. (2013)).

Stationary point conditions for the action are $\delta J=0 . \delta J / \delta \mathbf{u}=0$ gives the Clebsch representation for $\mathbf{u}$ :

$$
\mathbf{u}=\nabla \phi-\frac{\beta}{\rho} \nabla S-\frac{\lambda}{\rho} \nabla \mu+\mathbf{u}_{M}
$$

where

$$
\mathbf{u}_{M}=-\frac{(\nabla \times \boldsymbol{\Gamma}) \times \mathbf{B}}{\rho}-\boldsymbol{\Gamma} \frac{\nabla \cdot \mathbf{B}}{\rho},
$$

is magnetic contribution to $\mathbf{u}$. Setting $\delta J / \delta \phi, \delta J / \delta \beta, \delta J / \delta \lambda, \delta J / \delta \boldsymbol{\Gamma}$ consecutively equal to zero gives the mass, entropy advection, Lin constraint, and Faraday (magnetic flux 
conservation) constraint equations:

$$
\begin{aligned}
& \rho_{t}+\nabla \cdot(\rho \mathbf{u})=0, \\
& S_{t}+\mathbf{u} \cdot \nabla S=0, \\
& \mu_{t}+\mathbf{u} \cdot \nabla \mu=0, \\
& \mathbf{B}_{t}-\nabla \times(\mathbf{u} \times \mathbf{B})+\mathbf{u}(\nabla \cdot \mathbf{B})=0 .
\end{aligned}
$$

Setting $\delta J / \delta \rho, \delta J / \delta S, \delta J / \delta \mu, \delta J / \delta \mathbf{B}$ equal to zero gives evolution equations for the Clebsch potentials $\phi, \beta, \lambda$ and $\boldsymbol{\Gamma}$ as:

$$
\begin{aligned}
& -\left(\frac{\partial \phi}{\partial t}+\mathbf{u} \cdot \nabla \phi\right)+\frac{1}{2} u^{2}-h=0, \\
& \frac{\partial \beta}{\partial t}+\nabla \cdot(\beta \mathbf{u})+\rho T=0, \\
& \frac{\partial \lambda}{\partial t}+\nabla \cdot(\lambda \mathbf{u})=0, \\
& \frac{\partial \boldsymbol{\Gamma}}{\partial t}-\mathbf{u} \times(\nabla \times \boldsymbol{\Gamma})+\nabla(\boldsymbol{\Gamma} \cdot \mathbf{u})+\frac{\mathbf{B}}{\mu_{0}}=0 .
\end{aligned}
$$

Equation (3.18) is related to Bernoulli's equation for potential flow.The $\nabla(\boldsymbol{\Gamma} \cdot \mathbf{u})$ term in (3.21) is associated with $\nabla \cdot \mathbf{B} \neq 0$. Taking the curl of (3.21) gives:

$$
\frac{\partial \tilde{\boldsymbol{\Gamma}}}{\partial t}-\nabla \times(\mathbf{u} \times \tilde{\boldsymbol{\Gamma}})=-\frac{\nabla \times \mathbf{B}}{\mu_{0}} \quad \text { where } \quad \tilde{\boldsymbol{\Gamma}}=\nabla \times \mathbf{\Gamma} .
$$

Equations (3.18)-(3.22) can be written in the form:

$$
\begin{aligned}
& \frac{d \phi}{d t}=\frac{1}{2} u^{2}-h, \quad \frac{d}{d t}\left(\frac{\beta}{\rho}\right)=-T, \\
& \frac{d}{d t}\left(\lambda d^{3} x\right)=0 \quad \text { or } \quad \frac{d}{d t}\left(\frac{\lambda}{\rho}\right)=0, \\
& \frac{d}{d t}(\boldsymbol{\Gamma} \cdot d \mathbf{x})=-\frac{\mathbf{B} \cdot d \mathbf{x}}{\mu_{0}}, \quad \frac{d}{d t}(\tilde{\boldsymbol{\Gamma}} \cdot d \mathbf{S})=-\mathbf{J} \cdot d \mathbf{S} .
\end{aligned}
$$

where $d / d t=\partial / \partial t+\mathbf{u} \cdot \nabla$, is the Lagrangian time derivative following the flow and $\mathbf{J}=\nabla \times \mathbf{B} / \mu_{0}$ is the current. $\boldsymbol{\Gamma} \cdot d \mathbf{x}$ is a 1 -form and $\tilde{\boldsymbol{\Gamma}} \cdot d \mathbf{S}$ is a 2 -form and $d \mathbf{S}$ is an area element.

Introduce the Hamiltonian functional:

$$
\mathcal{H}=\int H d^{3} x \quad \text { where } \quad H=\frac{1}{2} \rho u^{2}+\epsilon(\rho, S)+\frac{B^{2}}{2 \mu_{0}} .
$$

Substitute the Clebsch expansion (3.15)-(3.16) for $\mathbf{u}$ in (3.24). Evaluating the variational derivatives of $\mathcal{H}$ gives Hamilton's equations:

$$
\begin{array}{llll}
\frac{\partial \rho}{\partial t}=\frac{\delta \mathcal{H}}{\delta \phi}, & \frac{\partial \phi}{\partial t}=-\frac{\delta \mathcal{H}}{\delta \rho}, & \frac{\partial S}{\partial t}=\frac{\delta \mathcal{H}}{\delta \beta}, & \frac{\partial \beta}{\partial t}=-\frac{\delta \mathcal{H}}{\delta S}, \\
\frac{\partial \mu}{\partial t}=\frac{\delta \mathcal{H}}{\delta \lambda}, & \frac{\partial \lambda}{\partial t}=-\frac{\delta \mathcal{H}}{\delta \mu}, & \frac{\partial \mathbf{B}}{\partial t}=\frac{\delta \mathcal{H}}{\delta \boldsymbol{\Gamma}}, & \frac{\partial \boldsymbol{\Gamma}}{\partial t}=-\frac{\delta \mathcal{H}}{\delta \mathbf{B}} .
\end{array}
$$

Here $\{\rho, \phi\},\{S, \beta\},\{\mu, \lambda\},\{\mathbf{B}, \boldsymbol{\Gamma}\}$ are canonically conjugate variables. 
The canonical Poisson bracket is:

$$
\begin{aligned}
\{F, G\}= & \int d^{3} x\left(\frac{\delta F}{\delta \rho} \frac{\delta G}{\delta \phi}-\frac{\delta F}{\delta \phi} \frac{\delta G}{\delta \rho}+\frac{\delta F}{\delta \mathbf{B}} \cdot \frac{\delta G}{\delta \boldsymbol{\Gamma}}-\frac{\delta F}{\delta \boldsymbol{\Gamma}} \cdot \frac{\delta G}{\delta \mathbf{B}}\right. \\
& \left.+\frac{\delta F}{\delta S} \frac{\delta G}{\delta \beta}-\frac{\delta F}{\delta \beta} \frac{\delta G}{\delta S}+\frac{\delta F}{\delta \mu} \frac{\delta G}{\delta \lambda}-\frac{\delta F}{\delta \lambda} \frac{\delta G}{\delta \mu}\right) .
\end{aligned}
$$

In terms of the Poisson bracket (3.26) the time evolution of a functional $F$ of the canonical or physical variables is given by $F_{t}=\{F, H\}$ where $H$ is the Hamiltonian of the system. The canonical Poisson bracket (3.26) satisfies the linearity, skew symmetry and Jacobi identity necessary for a Hamiltonian system (i.e. the Poisson bracket defines a Lie algebra).

Remark Cotter et al. (2007) derive the Clebsch variable equations analogous to (3.15)(3.22), by using an advected or Lie dragging formalism, in which the advected quantity $a$ satisfies the Lie dragging equation:

$$
\left(\frac{\partial}{\partial t}+\mathcal{L}_{\mathbf{u}}\right) a=0
$$

where $\mathcal{L}_{\mathbf{u}}$ is the Lie derivative of $a$ with respect to the vector field $\mathbf{u}$. Examples of Lie dragged quantities are $\omega^{0}=S$ (a scalar or 0 -form), $\omega^{2}=\mathbf{B} \cdot d \mathbf{S}$ is the Faraday 2-form, and $\omega^{3}=\rho d^{3} x$ is the mass 3 -form (see also Webb et al. (2014a) for further description of Lie dragged invariants in MHD). They also introduce fluid labels $\mathbf{l}^{A}$ which are advected with the flow, which are useful in specifying the initial state of the fluid, and their canonically conjugate momenta $\pi_{A}$ which are the Lagrange multipliers for $\mathbf{l}^{A}$ in the variational principle. They show how the Clebsch variational equations, the momentum

map (Clebsch variational equation obtained by varying $\mathbf{u}$ ) can be combined to yield the Euler-Poincaré or Eulerian momentum equation for the system.

\subsection{Non-Canonical Poisson Brackets}

Morrison and Greene $(1980,1982)$ introduced non-canonical Poisson brackets for MHD. Morrison and Greene (1980) gave the non-canonical Poisson bracket for MHD for the case $\nabla \cdot \mathbf{B}=0$. Morrison and Greene (1982) gave the form of the Poisson bracket for $\nabla \cdot \mathbf{B} \neq 0$. A detailed discussion of the non-canonical Poisson bracket and the Jacobi identity is given by Morrison (1982). Holm and Kupershmidt $(\mathbf{1 9 8 3 a}, \mathrm{b})$ point out that their Poisson bracket has the form expected for a semi-direct product Lie algebra, for which the Jacobi identity is automatically satisfied. Chandre et al. (2013) use Dirac's theory of constraints to derive properties of the Poisson bracket for the case $\nabla \cdot \mathbf{B}=0$.

Introduce the new variables:

$$
\mathbf{M}=\rho \mathbf{u}, \quad \sigma=\rho S,
$$

noting that

$$
\mathbf{M}=\rho \mathbf{u}=\rho \nabla \phi-\beta \nabla S-\lambda \nabla \mu+\mathbf{B} \cdot(\nabla \boldsymbol{\Gamma})^{T}-\mathbf{B} \cdot \nabla \boldsymbol{\Gamma}-\boldsymbol{\Gamma}(\nabla \cdot \mathbf{B}),
$$

and transforming the canonical Poisson bracket (3.26) from the old variables $(\rho, \phi, S, \beta, \mathbf{B}, \boldsymbol{\Gamma})$ to the new variables $(\rho, \sigma, \mathbf{B}, \mathbf{M})$ we obtain the Morrison and Greene (1982) non-canonical 
Poisson bracket:

$$
\begin{aligned}
\{F, G\}= & -\int d^{3} x\left\{\rho\left[\frac{\delta F}{\delta \mathbf{M}} \cdot \nabla\left(\frac{\delta G}{\delta \rho}\right)-\frac{\delta G}{\delta \mathbf{M}} \cdot \nabla\left(\frac{\delta F}{\delta \rho}\right)\right]\right. \\
& +\sigma\left[\frac{\delta F}{\delta \mathbf{M}} \cdot \nabla\left(\frac{\delta G}{\delta \sigma}\right)-\frac{\delta G}{\delta \mathbf{M}} \cdot \nabla\left(\frac{\delta F}{\delta \sigma}\right)\right] \\
& +\mathbf{M} \cdot\left[\left(\frac{\delta F}{\delta \mathbf{M}} \cdot \nabla\right) \frac{\delta G}{\delta \mathbf{M}}-\left(\frac{\delta G}{\delta \mathbf{M}} \cdot \nabla\right) \frac{\delta F}{\delta \mathbf{M}}\right] \\
& +\mathbf{B} \cdot\left[\frac{\delta F}{\delta \mathbf{M}} \cdot \nabla\left(\frac{\delta G}{\delta \mathbf{B}}\right)-\frac{\delta G}{\delta \mathbf{M}} \cdot \nabla\left(\frac{\delta F}{\delta \mathbf{B}}\right)\right] \\
& \left.+\mathbf{B} \cdot\left[\left(\nabla \frac{\delta F}{\delta \mathbf{M}}\right) \cdot \frac{\delta G}{\delta \mathbf{B}}-\left(\nabla \frac{\delta G}{\delta \mathbf{M}}\right) \cdot \frac{\delta F}{\delta \mathbf{B}}\right]\right\} .
\end{aligned}
$$

The bracket (3.30) has the Lie-Poisson form and satisfies the Jacobi identity for all functionals $F$ and $G$ of the physical variables, and in general applies both for $\nabla \cdot \mathbf{B} \neq 0$ and $\nabla \cdot \mathbf{B}=0$.

\subsubsection{Advected A Formulation}

Consider the MHD variational principle using the magnetic vector potential $\mathbf{A}$ instead of using $\mathbf{B}$ (e.g. Holm and Kupershmidt (1983a,b)). The condition that the magnetic flux $\mathbf{B} \cdot d \mathbf{S}$ is Lie dragged with the flow (i.e. Faraday's equation) as a constraint equation, is satisfied if the magnetic vector potential 1-form $\alpha=\mathbf{A} \cdot d \mathbf{x}$ is Lie dragged by the flow, where $\mathbf{B}=\nabla \times \mathbf{A}$. The condition that the one-form $\alpha=\mathbf{A} \cdot d \mathbf{x}$ is Lie dragged with the flow implies:

$$
\frac{\partial \mathbf{A}}{\partial t}-\mathbf{u} \times(\nabla \times \mathbf{A})+\nabla(\mathbf{u} \cdot \mathbf{A})=0
$$

(see Gordin and Petviashvili (1987), Padhye and Morrison (1996a,b), Webb et al. (2014a)). The condition that the magnetic flux $\beta=d \alpha=\mathbf{B} \cdot d \mathbf{S}$ is Lie dragged with the flow implies Faraday's equation:

$$
\frac{\partial \mathbf{B}}{\partial t}-\nabla \times(\mathbf{u} \times \mathbf{B})+\mathbf{u} \nabla \cdot \mathbf{B}=0
$$

for the magnetic induction $\mathbf{B}$. Note that the curl of (3.31) with $\mathbf{B}=\nabla \times \mathbf{A}$ gives Faraday's equation (3.32) where $\nabla \cdot \mathbf{B}=0$.

We use the variational principle $\delta \mathcal{A}=0$ where the action $\mathcal{A}$ is given by:

$$
\begin{aligned}
\mathcal{A}= & \int_{V} d^{3} x \int d t\left\{\left[\frac{1}{2} \rho|\mathbf{u}|^{2}-\varepsilon(\rho, S)-\frac{|\nabla \times \mathbf{A}|^{2}}{2 \mu_{0}}\right]\right. \\
& +\phi\left(\frac{\partial \rho}{\partial t}+\nabla \cdot(\rho \mathbf{u})\right)+\beta\left(\frac{\partial S}{\partial t}+\mathbf{u} \cdot \nabla S\right)+\lambda\left(\frac{\partial \mu}{\partial t}+\mathbf{u} \cdot \nabla \mu\right) \\
& \left.+\gamma \cdot\left[\frac{\partial \mathbf{A}}{\partial t}-\mathbf{u} \times(\nabla \times \mathbf{A})+\nabla(\mathbf{u} \cdot \mathbf{A})\right]\right\} .
\end{aligned}
$$

By setting the variational derivative $\delta \mathcal{A} / \delta \mathbf{u}=0$ gives the Clebsch variable expansion:

$$
\mathbf{u}=\nabla \phi-\frac{\beta}{\rho} \nabla S-\frac{\lambda}{\rho} \nabla \mu-\frac{\gamma \times(\nabla \times \mathbf{A})}{\rho}+\frac{\nabla \cdot \boldsymbol{\gamma}}{\rho} \mathbf{A}
$$

for the fluid velocity $\mathbf{u}$.

Setting the variational derivatives $\delta \mathcal{A} / \delta \phi, \delta \mathcal{A} / \delta \beta, \delta \mathcal{A} / \delta \lambda, \delta \mathcal{A} / \delta \boldsymbol{\gamma}$ equal to zero gives 
the constraint equations:

$$
\begin{aligned}
& \frac{\partial \rho}{\partial t}+\nabla \cdot(\rho \mathbf{u})=0, \quad \frac{\partial S}{\partial t}+\mathbf{u} \cdot \nabla S=0, \\
& \frac{\partial \mu}{\partial t}+\mathbf{u} \cdot \nabla \mu=0, \\
& \frac{\partial \mathbf{A}}{\partial t}-\mathbf{u} \times(\nabla \times \mathbf{A})+\nabla(\mathbf{u} \cdot \mathbf{A})=0 .
\end{aligned}
$$

Similarly setting $\delta \mathcal{A} / \delta \rho, \delta \mathcal{A} / \delta S, \delta \mathcal{A} / \delta \mu$ and $\delta \mathcal{A} / \delta \mathbf{A}$ equal to zero gives the equations:

$$
\begin{aligned}
& \frac{\partial \phi}{\partial t}+\mathbf{u} \cdot \nabla \phi+h-\frac{1}{2}|\mathbf{u}|^{2}=0 \\
& \frac{\partial \beta}{\partial t}+\nabla \cdot(\beta \mathbf{u})+\rho T=0, \quad \frac{\partial \lambda}{\partial t}+\nabla \cdot(\lambda \mathbf{u})=0 \\
& \frac{\partial \gamma}{\partial t}-\nabla \times(\mathbf{u} \times \gamma)+\mathbf{u}(\nabla \cdot \gamma)+\frac{\nabla \times \mathbf{B}}{\mu}=0 .
\end{aligned}
$$

The Euler-Lagrange equations (3.34)-(3.36) together imply Hamilton's equations:

$$
\begin{array}{llll}
\frac{\partial \rho}{\partial t}=\frac{\delta \mathcal{H}}{\delta \phi}, & \frac{\partial \phi}{\partial t}=-\frac{\delta \mathcal{H}}{\delta \rho}, & \frac{\partial S}{\partial t}=\frac{\delta \mathcal{H}}{\delta \beta}, & \frac{\partial \beta}{\partial t}=-\frac{\delta \mathcal{H}}{\delta S}, \\
\frac{\partial \mu}{\partial t}=\frac{\delta \mathcal{H}}{\delta \lambda}, & \frac{\partial \lambda}{\partial t}=-\frac{\delta \mathcal{H}}{\delta \mu}, & \frac{\partial \mathbf{A}}{\partial t}=\frac{\delta \mathcal{H}}{\delta \boldsymbol{\gamma}}, & \frac{\partial \boldsymbol{\gamma}}{\partial t}=-\frac{\delta \mathcal{H}}{\delta \mathbf{A}} .
\end{array}
$$

Here $\{\rho, \phi\},\{S, \beta\}$, and $\{\mathbf{A}, \gamma\}$ are canonically conjugate variables. The Hamiltonian functional $\mathcal{H}$ is given by (3.24), and $\mathbf{u}$ is given by the Clebsch expansion (3.34). The canonical Poisson bracket is:

$$
\begin{aligned}
\{F, G\}= & \int d^{3} x\left(\frac{\delta F}{\delta \rho} \frac{\delta G}{\delta \phi}-\frac{\delta F}{\delta \phi} \frac{\delta G}{\delta \rho}+\frac{\delta F}{\delta \mathbf{A}} \cdot \frac{\delta G}{\delta \gamma}-\frac{\delta F}{\delta \gamma} \cdot \frac{\delta G}{\delta \mathbf{A}}\right. \\
& \left.+\frac{\delta F}{\delta S} \frac{\delta G}{\delta \beta}-\frac{\delta F}{\delta \beta} \frac{\delta G}{\delta S}+\frac{\delta F}{\delta \mu} \frac{\delta G}{\delta \lambda}-\frac{\delta F}{\delta \lambda} \frac{\delta G}{\delta \mu}\right)
\end{aligned}
$$

The transformations of the variational derivatives from canonical Clebsch variables $(\rho, \phi, S, \beta, \mathbf{A}, \gamma)$ in terms of the non-canonical new variables $(\rho, \sigma, \mathbf{A}, \mathbf{M})$ are:

$$
\begin{aligned}
& \frac{\delta F}{\delta \rho}=\frac{\delta F}{\delta \rho}+S \frac{\delta F}{\delta \sigma}+\frac{\delta F}{\delta \mathbf{M}} \cdot \nabla \phi, \quad \frac{\delta F}{\delta \phi}=-\nabla \cdot\left(\rho \frac{\delta F}{\delta \mathbf{M}}\right) \\
& \frac{\delta F}{\delta S}=\rho \frac{\delta F}{\delta \sigma}+\nabla \cdot\left(\beta \frac{\delta F}{\delta \mathbf{M}}\right), \quad \frac{\delta F}{\delta \beta}=-\frac{\delta F}{\delta \mathbf{M}} \cdot \nabla S \\
& \frac{\delta F}{\delta \mathbf{A}}=\frac{\delta F}{\delta \mathbf{A}}+\nabla \cdot \gamma \frac{\delta F}{\delta \mathbf{M}}-\nabla \times\left(\frac{\delta F}{\delta \mathbf{M}} \times \gamma\right) \\
& \frac{\delta F}{\delta \gamma}=-\mathbf{B} \times \frac{\delta F}{\delta \mathbf{M}}-\nabla\left[\mathbf{A} \cdot\left(\frac{\delta F}{\delta \mathbf{M}}\right)\right] \\
& \frac{\delta F}{\delta \mu}=\nabla \cdot\left(\lambda \frac{\delta F}{\delta \mathbf{M}}\right), \quad \frac{\delta F}{\delta \lambda}=-\frac{\delta F}{\delta \mathbf{M}} \cdot \nabla \mu .
\end{aligned}
$$

In terms of the non-canonical variables $(\mathbf{M}, \mathbf{A}, \rho, \sigma)$ where $\sigma=\rho S$ we obtain the 
non-canonical Poisson bracket:

$$
\begin{aligned}
\{F, G\}= & -\int d^{3} x\left\{\left[F_{\mathbf{M}} \cdot \nabla\left(G_{\mathbf{M}}\right)-G_{\mathbf{M}} \cdot \nabla\left(F_{\mathbf{M}}\right)\right] \cdot \mathbf{M}\right. \\
& +\rho\left[F_{\mathbf{M}} \cdot \nabla\left(G_{\rho}\right)-G_{\mathbf{M}} \cdot \nabla\left(F_{\rho}\right)\right] \\
& +\sigma\left[F_{\mathbf{M}} \cdot \nabla\left(G_{\sigma}\right)-G_{\mathbf{M}} \cdot \nabla\left(F_{\sigma}\right)\right] \\
& +\mathbf{A} \cdot\left[F_{\mathbf{M}} \nabla \cdot\left(G_{\mathbf{A}}\right)-G_{\mathbf{M}} \nabla \cdot\left(F_{\mathbf{A}}\right)\right] \\
& \left.+\nabla \times \mathbf{A} \cdot\left[G_{\mathbf{A}} \times F_{\mathbf{M}}-F_{\mathbf{A}} \times G_{\mathbf{M}}\right]\right\},
\end{aligned}
$$

where $F_{\mathbf{M}} \equiv \delta F / \delta \mathbf{M}$ and similarly for the other variational derivatives in (3.40). The noncanonical bracket (3.40) was obtained by Holm and Kupershmidt (1983a,b). It is a skew symmetric bracket and satisfies the Jacobi identity. Holm and Kupershmidt (1983a,b) show that bracket (3.40) corresponds to a semi-direct product Lie algebra.

\section{Overview of Multi-symplectic Systems}

In this section we discuss multi-symplectic systems of partial differential equations. In Section 4.1, we set out the general theory, for the case where the space-time metric is flat. We assume the independent variables $x^{\alpha}$ have a flat metric. For example in MHD we take the independent variables as $(t, x, y, z)$ where $(x, y, z)$ are Cartesian space coordinates and $t$ is the time. In Section 4.2 we indicate how the analysis is altered if the independent variables are generalized coordinates (e.g. for spherical or cylindicral symmetry the metric plays a role via the replacement of ordinary partial derivatives by co-variant derivatives). Bridges et al. (2010) also describe the general case of multisymplectic systems taking into account the geometry of the independent variables. They use the algebra of exterior differential forms and the variational bi-complex, in which the exterior differential $d=d_{h}+d_{v}$ where $d_{h}$ is the so-called horizontal exterior derivative and $d_{v}$ is the vertical exterior derivative.

\subsection{Flat Cartesian metric}

Hamiltonian systems, with one evolution variable $t$, can in general be written in the form:

$$
\mathrm{K}_{i j}(z) \frac{d z^{j}}{d t}=\nabla_{z^{i}} H(z)
$$

where the invariant phase space volume element:

$$
\kappa=\frac{1}{2} \mathrm{~K}_{i j}(z) d z^{i} \wedge d z^{j}
$$

is a closed two-form, i.e. $d \kappa=0$. Here $d$ denotes the exterior derivative and $\wedge$ denotes the anti-symmetric wedge product used in the exterior Calculus. The condition that $\kappa$ be a closed 2-form, implies by the Poincaré Lemma, that $\kappa=d g$ where $g=L_{j} d z^{j}$ is a one-form (note that $d \kappa=d d g=0$ by antisymmetry of the wedge product). It turns out, that the condition that $\kappa$ be a closed 2-form implies that $\mathrm{K}_{i j}=-\mathrm{K}_{j i}$ is a skew symmetric operator (see Zakharov and Kuznetsov (1997), and Hydon (2005)). Taking the exterior derivative of the 2 -form (4.2) and setting the result equal to zero, we obtain the identity:

$$
\mathrm{K}_{i j, k}+\mathrm{K}_{j k, i}+\mathrm{K}_{k i, j}=0,
$$

which in some cases is related to the Jacobi identity for the Poisson bracket. If the system (4.1) has an even dimension, and if $\mathrm{K}_{i j}$ has non-zero determinant, then (4.1) can 
be written in the form:

$$
\frac{d z^{i}}{d t}=\mathrm{R}_{i j} \nabla_{z^{j}} H(z)
$$

where $\mathrm{R}_{i j}$ is the inverse of the matrix $\mathrm{K}_{i j}$. Here $\mathrm{R}_{i j}=-\mathrm{R}_{j i}$ is a skew-symmetric matrix. The closure relation (4.3) then are equivalent to the relations:

$$
\mathrm{R}_{i m} \frac{\partial \mathrm{R}_{j k}}{\partial z^{m}}+\mathrm{R}_{k m} \frac{\partial \mathrm{R}_{i j}}{\partial z^{m}}+\mathrm{R}_{j m} \frac{\partial \mathrm{R}_{k i}}{\partial z^{m}}=0,
$$

(see e.g. Zakharov and Kuznetsov (1997)). The Poisson bracket for the system in the finite dimensional case is given by

$$
\{A, B\}=\sum \mathrm{R}_{i j} \frac{\partial A}{\partial z^{i}} \frac{\partial B}{\partial z^{j}}
$$

Using the Poisson bracket description (4.6) the Jacobi identity reduces to (4.5). Casimir functions or more generally functionals have zero Poisson bracket with respect to any other functional of the variables describing the system. For finite dimensional systems Casimirs always occur for odd dimensional systems.

A finite dimensional Hamiltonian system of dimension $2 n$ with canonical variables $z=\left(q^{1}, q^{2}, \ldots q^{n}, p_{1}, p_{2}, \ldots p_{n}\right)^{t}$ can be written in the form (4.1), where

$$
\mathrm{K}=\mathrm{J}^{t}=\left(\begin{array}{cc}
0 & -I_{n} \\
I_{n} & 0
\end{array}\right)
$$

Here the matrix $\mathrm{K}$ is the inverse of the symplectic matrix $\mathrm{J}$ and $I_{n}$ is the unit $n \times n$ matrix. The invariant phase space element form (4.2) is:

$$
\kappa=d p_{j} \wedge d q^{j}=d\left(p_{j} d q^{j}\right) .
$$

Hamiltonian, multi-symplectic systems with $n$ independent variables $x^{\alpha}$ can be written in the form:

$$
\mathrm{K}_{i j}^{\alpha} z_{, \alpha}^{j}=\nabla_{z^{i}} H(z)
$$

where $z_{\alpha}^{j}=\partial z^{j} / \partial x^{\alpha}$. The fundamental invariant 2-forms are:

$$
\kappa^{\alpha}=\frac{1}{2} \mathrm{~K}_{i j}^{\alpha} d z^{i} \wedge d z^{j}, \quad \alpha=1(1) n,
$$

Invariance of the phase space element $D_{t}\left(d p_{j} \wedge d q^{j}\right)=0$ for the standard canonical Hamiltonian formulation with evolution variable $t$ is replaced by the symplectic, or structural conservation law:

$$
\kappa_{, \alpha}^{\alpha}=0
$$

which is referred to as the symplecticity conservation law.

The closure of the 2-forms $\kappa^{\alpha}$ implies that the exterior derivative of $\kappa^{\alpha}=0$. By the Poincaré Lemma $\kappa^{\alpha}$ is the exterior derivative of a 1 -form, i.e.,

$$
\kappa^{\alpha}=d\left(L_{j}^{\alpha} d z^{j}\right)=d \omega^{\alpha} \quad \text { where } \omega^{\alpha}=L_{j}^{\alpha} d z^{j} .
$$

Note that $d \kappa^{\alpha}=d d \omega^{\alpha}=0$. Taking the exterior derivative of $\omega^{\alpha}$ in (4.12) and using the anti-symmetry of the wedge product we obtain:

$$
\kappa^{\alpha}=\frac{1}{2}\left(\frac{\partial L_{k}^{\alpha}}{\partial z^{j}}-\frac{\partial L_{j}^{\alpha}}{\partial z^{k}}\right) d z^{j} \wedge d z^{k}
$$


From (4.10) and (4.13) we obtain:

$$
\mathrm{K}_{j k}^{\alpha}=\frac{\partial L_{k}^{\alpha}}{\partial z^{j}}-\frac{\partial L_{j}^{\alpha}}{\partial z^{k}}
$$

Thus, the matrices $\mathrm{K}_{i j}^{\alpha}$ are skew-symmetric, i.e. $\mathrm{K}_{i j}^{\alpha}=-\mathrm{K}_{j i}^{\alpha}$.

Proposition 4.1. The Legendre transformation for multi-symplectic systems is the identity

$$
\left(L_{j}^{\alpha} d z^{j}\right)_{, \alpha}=d\left\{L_{j}^{\alpha}(z) z_{, \alpha}^{j}-H(z)\right\} \equiv d L,
$$

where

$$
L=L_{j}^{\alpha}(z) z_{, \alpha}^{j}-H(z),
$$

is the Lagrangian density and $H(z)$ is the multi-symplectic Hamiltonian.

Proof.The proof of (4.15) proceeds by noting

$$
\begin{aligned}
\left(L_{j}^{\alpha} d z^{j}\right)_{, \alpha} & =\frac{\partial L_{j}^{\alpha}}{\partial z^{i}} z_{, \alpha}^{i} d z^{j}+L_{j}^{\alpha}(z) D_{\alpha} d z^{j} \\
& =\frac{\partial L_{j}^{\alpha}}{\partial z^{i}} z_{, \alpha}^{i} d z^{j}+L_{j}^{\alpha}(z) d\left(z_{, \alpha}^{j}\right) .
\end{aligned}
$$

Here we used the fact that the operators $d$ and $D_{\alpha}$ commute. (4.17) can be further reduced to:

$$
\left(L_{j}^{\alpha} d z^{j}\right)_{, \alpha}=-\mathrm{K}_{j i}^{\alpha} z_{, \alpha}^{i} d z^{j}+d\left(L_{j}^{\alpha}(z) z_{, \alpha}^{j}\right) .
$$

The identity (4.15) then follows by using the Hamiltonian evolution equations (4.9).

The symplecticity or structural conservation law (4.11) now follows by taking the exterior derivative of (4.15) and using the results $d d L=0$ and $d D_{\alpha}=D_{\alpha} d$, i.e.,

$$
D_{\alpha} \kappa^{\alpha}=D_{\alpha}\left[d\left(L_{j}^{\alpha} d z^{j}\right)\right]=d D_{\alpha}\left(L_{j}^{\alpha} d z^{j}\right)=d d L=0,
$$

which is (4.11). Other conservation laws are obtained by sectioning the forms in (4.15) (i.e. we impose the requirement that $z^{j}=z^{j}(\mathbf{x})$, which is also referred to as the pull-back to the base manifold). The pullback, applied to (4.15) gives

$$
\left(L_{j}^{\alpha} d z^{j}\right)_{, \alpha}=\left(L_{j}^{\alpha} z_{, \beta}^{j} d x^{\beta}\right)_{, \alpha}=\left(L_{j}^{\alpha} z_{, \beta}^{j}\right)_{, \alpha} d x^{\beta}=d L=\frac{\partial L}{\partial x^{\beta}} d x^{\beta} .
$$

Thus, (4.20) gives the conservation law:

$$
D_{\alpha}\left(L_{j}^{\alpha}(z) z_{, \beta}^{j}-L \delta_{\beta}^{\alpha}\right)=0 .
$$

This conservation law is in fact, the conservation law obtained due to the invariance of the action $A=\int L d x$ under translations in $x^{\beta}$ which follows from Noether's first theorem (i.e. $x^{\alpha}=x^{\alpha}+\epsilon \delta_{\beta}^{\alpha}$ ).

A further set of $n(n-1) / 2$ conservation laws is obtained from pull-back of the structural conservation law (4.11) to the base manifold, namely:

$$
D_{\alpha}\left(\mathrm{K}_{i j}^{\alpha} z_{, \beta}^{i} z_{, \gamma}^{j}\right)=0, \quad \beta<\gamma .
$$

The conservation laws (4.22) can be obtained by cross-differentiation of the conservation laws (4.19), i.e. they are a consequence of the equations:

$$
D_{\gamma} G_{\beta}-D_{\beta} G_{\gamma}=D_{\alpha}\left(\mathrm{K}_{i j}^{\alpha} z_{, \gamma}^{i} z_{, \beta}^{j}\right)
$$


where

$$
G_{\beta}=D_{\alpha}\left(L_{j}^{\alpha} z_{, \beta}^{j}-L \delta_{\beta}^{\alpha}\right) .
$$

Here $G_{\beta}=0$ give the energy and momentum conservation equations for $\beta=$ $0,1,2,3$ respectively. A multi-symplectic version of Noether's theorem for the multisymplectic system (4.9) is described below (see also Hydon (2005)):

Proposition 4.2. If the action:

$$
J=\int L d^{3} x d t
$$

is invariant to $O(\epsilon)$ under the infinitesimal Lie transformation:

$$
z^{\prime} s=z^{s}+\epsilon V^{z^{s}}, \quad x^{\prime \alpha}=x^{\alpha}+\epsilon V^{x^{\alpha}}, \quad(0 \leqslant \alpha \leqslant 3, \quad 1 \leqslant s \leqslant N),
$$

and under the divergence transformation:

$$
L^{\prime}=L+\epsilon D_{\alpha} \Lambda^{\alpha}+O\left(\epsilon^{2}\right)
$$

where $L$ has the multi-symplectic form (4.16):

$$
L=L_{j}^{\alpha}(z) z_{, \alpha}^{j}-H(z) \quad \text { and } \quad \mathcal{H}=\int H(z) d^{3} x d t,
$$

is the Hamiltonian functional, then the Euler Lagrange equations for the action:

$$
E_{z^{s}}(L)=\frac{\partial L}{\partial z^{s}}-\frac{\partial}{\partial x^{\alpha}}\left(\frac{\partial L}{\partial z_{, \alpha}^{s}}\right) \equiv-\frac{\partial H}{\partial z^{s}}+\mathrm{K}_{s j}^{\alpha} z_{, \alpha}^{j}=0
$$

admit the conservation law

$$
D_{\alpha}\left\{V^{x^{\alpha}} L+W^{\alpha}\left[\mathbf{z}, \hat{V}^{\mathbf{z}}\right]+\Lambda^{\alpha}\right\}=0 .
$$

$\operatorname{In}(4.30)$

$$
W^{\alpha}\left[\mathbf{z}, \hat{V}^{\mathbf{z}}\right]=\hat{V}^{z^{s}} \frac{\partial L}{\partial z_{, \alpha}^{s}} \equiv \hat{V}^{z^{s}} L_{s}^{\alpha}(z)
$$

and

$$
\hat{V}^{z^{s}}=V^{z^{s}}-V^{x^{\alpha}} z_{, \alpha}^{s},
$$

is the canonical or characteristic Lie symmetry generator (i.e., the infinitesimal Lie symmetry transformation $z^{\prime s}=z^{s}+\epsilon \hat{V}^{z^{s}}, x^{\alpha}=x^{\alpha}$ which is equivalent to Lie transformation (4.26)). Thus, the conservation law (4.30) reduces to:

$$
D_{\alpha}\left\{V^{x^{\alpha}}\left[L_{s}^{\mu}(\mathbf{z}) z_{, \mu}^{s}-H(\mathbf{z})\right]+\hat{V}^{z^{s}} L_{s}^{\alpha}(\mathbf{z})+\Lambda^{\alpha}\right\}=0 .
$$

or aternatively:

$$
D_{\alpha}\left\{V^{x^{\alpha}} L+\hat{V}^{z^{s}} L_{s}^{\alpha}(\mathbf{z})+\Lambda^{\alpha}\right\}=0 .
$$

This is the multi-symplectic form of Noether's first theorem for the system (4.9).

The condition for the Lie symmetry (4.26)-(4.27) to be a divergence symmetry of the action is:

$$
\tilde{X} L+V^{x^{\alpha}} D_{\alpha} L+D_{\alpha} \Lambda^{\alpha}=0,
$$

where

$$
\tilde{X}=V^{x^{\alpha}} \frac{\partial}{\partial x^{\alpha}}+V^{z^{s}} \frac{\partial}{\partial z^{s}}+V_{z_{,}^{s}}^{z_{2}} \frac{\partial}{\partial z_{, \alpha}^{s}}+\ldots
$$


is the extended Lie symmetry operator. The extended Lie symmetry operator $\tilde{X}$ can be expressed in terms of the characteristic symmetry operator $\hat{X}$ by the formula

$$
\tilde{X}=\hat{X}+V^{x^{\alpha}} D_{\alpha}, \quad \text { where } \quad \hat{X}=\hat{V}^{z^{s}} \frac{\partial}{\partial z^{s}}+D_{\alpha}\left(\hat{V}^{z^{s}}\right) \frac{\partial}{\partial z_{, \alpha}^{s}}+\ldots
$$

The Lie invariance condition (4.35) written in terms of $\hat{X}$ is:

$$
\hat{X} L+D_{\alpha}\left(V^{x^{\alpha}} L+\Lambda^{\alpha}\right)=0 .
$$

Example As an example of Noether's theorem, consider the invariance of the action $J$ under the Lie symmetry:

$$
V^{x^{\alpha}}=\delta_{\beta}^{\alpha}, \quad V^{z^{s}}=0, \quad \Lambda^{\alpha}=0,
$$

corresponding to translation invariance with respect to $x^{\beta}$. The canonical Lie symmetry generator $\hat{V}^{z^{s}}$ is given by:

$$
\hat{V}^{z^{s}}=-z_{, \beta}^{s},
$$

The Lie invariance condition (4.38) is satisfied for $\Lambda^{\alpha}=0$, i.e. the action is invariant under a variational symmetry (one can show $\hat{X} L=-D_{\beta} L$ ). The conservation law (4.33) or (4.34) reduces to the symplectic conservation law (4.21). Thus we have shown that the symplectic conservation law is due to invariance of the action under translations in $x^{\beta}$

Proposition 4.3. The multi-symplectic system (4.9) for the case where $x^{\alpha}=(t, x, y, z)$ is equivalent to the differential form system:

$$
\begin{aligned}
\Omega= & d \omega^{0} \wedge d x \wedge d y \wedge d z-d \omega^{1} \wedge d x^{0} \wedge d y \wedge d z+d \omega^{2} \wedge d x^{0} \wedge d x \wedge d z \\
& -d \omega^{3} \wedge d x^{0} \wedge d x \wedge d y-d H \wedge d x^{0} \wedge d x \wedge d y \wedge d z=0
\end{aligned}
$$

in which the $z^{\mu}$ are the dependent variables and the $x^{\alpha}$ are the independent variables. The variational principle

$$
J=\int \Omega
$$

with $\delta J / \delta z^{\mu}=0$ gives the multi-symplectic system (4.9). The form (4.41) is referred to as the Cartan-Poincaré form (e.g. Marsden and Shkoller (1999)).

Proof. Starting from (4.9) we require:

$$
\left(\mathrm{K}_{\mu \nu}^{\alpha} \frac{\partial z^{\nu}}{\partial x^{\alpha}}-\frac{\delta H}{\delta z^{\mu}}\right) d z^{\mu} \wedge d x^{0} \wedge d x \wedge d y \wedge d z=0 .
$$

Equation (4.43) can be expanded to give (4.41). In the derivation of (4.43) the forms are sectioned, i.e. the variables $z^{\mu}$ are taken to be dependent on the independent variables $x^{\alpha}, \alpha=0,1,2,3$.

Next we consider the variation of $\delta J$ in (4.42), which may be reduced to the form:

where

$$
\delta J=\int d\left(\delta z^{\mu}\right) \wedge \beta_{\mu}+\int \delta z^{\mu} d \beta_{\mu}
$$

$\beta_{\mu}=(-1)^{\alpha}\left[\mathrm{K}_{\mu \nu}^{\alpha} d z^{\nu} \wedge \prod_{j \neq \alpha} d x^{j}\right]-\frac{\partial H}{\partial z^{\mu}} \wedge \prod_{j=0}^{n} d x^{j}$,

$\prod_{j=0}^{n} d x^{j}=d x^{0} \wedge d x^{1} \ldots \wedge d x^{n}$ and $\prod_{j \neq \alpha} d x^{j}$ is defined similarly, but does not 
include $d x^{\alpha}$ in the wedge product. $n$ is the number of space variables. In the derivation of (4.44) we used the representation:

$$
\Omega=(-1)^{\alpha} K_{\mu \nu}^{\alpha} d z^{\mu} \wedge d z^{\nu} \wedge \prod_{j \neq \alpha} d x^{j}-\frac{\partial H}{\partial z^{\mu}} d z^{\mu} \wedge \prod_{j=0}^{n} d x^{j} \equiv d z^{\mu} \wedge \beta_{\mu},
$$

where we choose $\mu<\nu$ in the first term in (4.46), but there is no restriction on $\nu$ in (4.45). The first term in (4.44) is due to the variations of $\delta d z^{\mu}$, whereas the second term is due to the variations $\delta \mathrm{K}_{\mu \nu}^{\alpha}$ and $\delta\left(\partial H / \partial z^{\mu}\right)$. The second integral in (4.44) vanishes by Stokes theorem:

$$
\int_{V} d \beta_{\mu}=\int_{\partial V} \beta_{\mu}=0
$$

where we assume that $\beta_{\mu}$ vanishes on the boundary $\partial V$. For independent variations of $\delta z^{\mu}$, the condition $\delta J=0$ gives the differential form system:

$$
\beta_{\mu}=0, \quad 1 \leqslant \mu \leqslant N
$$

where $N$ is the number of dependent variables $z^{\mu}$.

Equations (4.48) form the basis of the Cartan differential form representation of the differential equation system (4.9). However, the ideal of forms representing the differential equation system (4.9) in general needs to be enlarged to include the exterior derivatives of $d \beta_{\mu}$ which are not expressible as a linear combination of the $\beta_{\mu}$. The closed system of forms consisting of the $\beta_{\mu}$ plus the adjoined forms $d \beta_{\nu}$ can then be used to represent the differential equation system (4.9) and the integrability conditions for the system (4.9) (see e.g. Harrison and Estabrook (1971)). This completes our discussion of the variational principle $(4.42)$.

\subsection{Covariant Formulation}

In Section 4.1 we assumed that that the $L_{j}^{\alpha}$ and the $\mathrm{K}_{i j}^{\alpha}$ depended only on the field variables z. Bridges et al. (2010) in a more general formulation using the variational bi-complex consider cases where the $L_{j}^{\alpha}$ and $H$ can also depend on the independent variables $x^{k}$. They show that the multi-symplectic system (4.9) is a special case of the more general system:

$$
\mathrm{K}_{i j}^{\alpha} \frac{\partial z^{j}}{\partial q^{\alpha}}-L_{i ; \alpha}^{\alpha}=\frac{\partial H}{\partial z^{i}}
$$

where

$$
L_{i ; \alpha}^{\alpha}=\frac{\partial L_{i}^{\alpha}}{\partial q^{\alpha}}+\Gamma_{s \alpha}^{\alpha} L_{i}^{s} \equiv \frac{1}{\sqrt{g}} \frac{\partial}{\partial q^{\alpha}}\left(\sqrt{g} L_{i}^{\alpha}\right),
$$

is the covariant derivative of the contravariant vector field $L_{i}^{\alpha}$ and $g=\operatorname{det}\left(g_{\alpha \beta}\right)$ is the determinant of the metric tensor where $d s^{2}=g_{\alpha \beta} d q^{\alpha} d q^{\beta}$ is the metric. Here $q^{\alpha}$ are generalized coordinates. The holonomic base vectors $\mathbf{e}_{\alpha}=\partial \mathbf{x} / \partial q^{\alpha}$ and $g_{\alpha \beta}=\mathbf{e}_{\alpha} \cdot \mathbf{e}_{\beta}$. In Section 4.1 we implicitly used Cartesian space time coordinates (i.e. $x^{\alpha}=(t, x, y, z)$ ). The differential equation system (4.49) also holds for generalized coordinates (e.g. for spherical polar or cylindrical space coordinates).

The system of equations (4.49)-(4.50) can be written in the form:

$$
\left(\frac{\partial L_{j}^{\alpha}}{\partial z^{i}}-\frac{\partial L_{i}^{\alpha}}{\partial z^{j}}\right) \frac{\partial z^{j}}{\partial q^{\alpha}}-\frac{1}{\sqrt{g}} \frac{\partial}{\partial q^{\alpha}}\left(\sqrt{g} L_{i}^{\alpha}\right)=\frac{\partial H}{\partial z^{i}},
$$


which highlights the fact that the system depends on the 1 -forms $\omega^{\alpha}=L_{j}^{\alpha} d z^{j}$, the Hamiltonian $H$ and the metric $g_{\alpha \beta}$.

Bridges et al. (2010) develop the theory of multi-symplectic systems of the form (4.49) and its relationship to the variational bi-complex (e.g. Anderson $(1989,1992)$; Bridges et al. (2010) and references therein).

Below we provide a discussion of the origin of (4.49). An alternative approach is to use the variational bi-complex analysis of Bridges et al. (2010).

Proposition 4.4. The multi-symplectic partial differential equation system (4.49) arises as the Euler Lagrange equation of the action:

$$
J=\iint L \sqrt{g} d^{3} q d t
$$

where

$$
L=L_{j}^{\alpha} z_{, \alpha}^{j}-H(z)
$$

is the Lagrangian in the original coordinates $(t, x, y, z)$. In particular, the stationary point conditions:

$$
E_{i}(\bar{L})=\sqrt{g}\left\{\mathrm{~K}_{i j}^{\alpha} z_{, \alpha}^{j}-L_{i ; \alpha}^{\alpha}-\frac{\partial H}{\partial z^{i}}\right\}=0,
$$

where $\bar{L}=L \sqrt{g}$, are equivalent to the multi-symplectic system (4.49).

Proof.Taking the variation of the functional (4.52) and integrating by parts gives:

$$
\begin{aligned}
\delta J= & \iint\left(\delta\left(L_{j}^{\alpha} \sqrt{g}\right) z_{, \alpha}^{j}+L_{j}^{\alpha} \sqrt{g} \delta z_{, \alpha}^{j}-\frac{\partial H}{\partial z^{s}} \delta z^{s} \sqrt{g}\right) d^{3} q d t \\
= & \iint \delta z^{j}\left(-\frac{1}{\sqrt{g}} D_{\alpha}\left(L_{j}^{\alpha} \sqrt{g}\right)-\frac{\partial H}{\partial z^{j}}+\frac{\partial L_{k}^{\alpha}}{\partial z^{j}} z_{, \alpha}^{k}\right) \sqrt{g} d^{3} q d t \\
& +\int d^{3} q \int d t D_{\alpha}\left(L_{j}^{\alpha} \sqrt{g} \delta z^{j}\right),
\end{aligned}
$$

where $D_{\alpha}$ denotes the total partial derivative with respect to $q^{\alpha}$. Noting that

$$
D_{\alpha}\left(L_{j}^{\alpha} \sqrt{g}\right)=\frac{\partial}{\partial q^{\alpha}}\left(L_{j}^{\alpha} \sqrt{g}\right)+\frac{\partial}{\partial z^{s}}\left(L_{j}^{\alpha} \sqrt{g}\right) z_{, \alpha}^{s},
$$

in $(4.55$ we obtain:

$$
\begin{aligned}
\delta J= & \iint \delta z^{j}\left\{\mathrm{~K}_{j s}^{\alpha} z_{, \alpha}^{s}-L_{j ; \alpha}^{\alpha}-\frac{\partial H}{\partial z^{s}}\right\} \sqrt{g} d^{3} q d t \\
& +\iint D_{\alpha}\left(L_{j}^{\alpha} \sqrt{g} \delta z^{j}\right) d^{3} q d t .
\end{aligned}
$$

Dropping the surface term in (4.57) and evaluating $\delta J / \delta z^{i}$ gives the Euler-Lagrange equation (4.54). This completes the proof.

Proposition 4.5. Let one-form $\omega^{\alpha}=L_{j}^{\alpha} d z^{j}$ be a contravariant vector field with respect to the index $\alpha$, then the multi-symplectic Legendre transformation (4.15)-(4.16) is replaced by the more general result:

$$
\left(L_{j}^{\alpha}(z ; x) d z^{j}\right)_{; \alpha}=d\left[L_{j}^{\alpha}(z ; x) z_{, \alpha}^{j}-H(z)\right]=d L,
$$

where

$$
L=L_{j}^{\alpha}(z ; x) z_{, \alpha}^{j}-H(z)
$$


is the Lagrangian density and $H(z)$ is the multi-symplectic Hamiltonian for the system (4.49), which satisfies the structural conservation law:

$$
\kappa_{; \alpha}^{\alpha}=0 \quad \text { where } \quad \kappa^{\alpha}=d \omega^{\alpha}=d\left(L_{j}^{\alpha} d z^{j}\right) .
$$

Proof. Computing the covariant derivative of $\omega^{\alpha}$ we obtain:

$$
\omega_{; \alpha}^{\alpha}=\left(L_{j}^{\alpha} d z^{j}\right)_{; \alpha}=L_{j ; \alpha}^{\alpha} d z^{j}+\left\{\frac{\partial L_{j}^{\alpha}}{\partial z^{k}} z_{, \alpha}^{k} d z^{j}+L_{j}^{\alpha} D_{\alpha}\left(d z^{j}\right)\right\},
$$

where the first term takes into account changes due to changes in $x^{\alpha}$ and the second term in curly brackets takes into account the changes in $z^{j}$ keeping $x^{\alpha}$ fixed. From (4.61) we obtain:

$$
\begin{aligned}
\omega_{; \alpha}^{\alpha} & =L_{j ; \alpha}^{\alpha} d z^{j}+\frac{\partial L_{j}^{\alpha}}{\partial z^{k}} z_{, \alpha}^{k} d z^{j}+L_{j}^{\alpha} d\left(z_{, \alpha}^{j}\right) \\
& =\left(L_{j ; \alpha}^{\alpha}+\frac{\partial L_{j}^{\alpha}}{\partial z^{k}} z_{, \alpha}^{k}\right) d z^{j}+\left\{d\left(L_{j}^{\alpha} z_{, \alpha}^{j}\right)-\frac{\partial L_{j}^{\alpha}}{\partial z^{k}} d z^{k} z_{, \alpha}^{j}\right\} \\
& =\left(\frac{\partial L_{j}^{\alpha}}{\partial z^{k}}-\frac{\partial L_{k}^{\alpha}}{\partial z^{j}}\right) z_{, \alpha}^{k} d z^{j}+L_{j ; \alpha}^{\alpha} d z^{j}+d\left(L_{j}^{\alpha} z_{, \alpha}^{j}\right) \\
& =\left(\mathrm{K}_{k j}^{\alpha} z_{, \alpha}^{k}+L_{j ; \alpha}^{\alpha}\right) d z^{j}+d(L+H) .
\end{aligned}
$$

From (4.62) we obtain:

$$
\omega_{; \alpha}^{\alpha}=-\left(\mathrm{K}_{j k}^{\alpha} z_{, \alpha}^{k}-L_{j ; \alpha}^{\alpha}-\frac{\partial H}{\partial z^{j}}\right) d z^{j}+d L .
$$

Using (4.49), (4.63) reduces to:

$$
\omega_{; \alpha}^{\alpha}=\left(L_{j}^{\alpha} d z^{j}\right)_{; \alpha}=d L .
$$

Taking the exterior derivative of (4.64) gives:

$$
d \omega_{; \alpha}^{\alpha}=D_{\alpha}\left(d \omega^{\alpha}\right)=D_{\alpha} \kappa^{\alpha}=d d L=0 .
$$

Thus, the differential equation system (4.49) is multi-symplectic, meaning $D_{\alpha} \kappa^{\alpha}=0$, where $\kappa^{\alpha}=d \omega^{\alpha}$ and $\omega^{\alpha}=L_{j}^{\alpha} d z^{j}$. This completes the proof.

The form of Noether's first theorem using the generalized coordinates $q^{\alpha}=\left(t, q^{1}, q^{2}, q^{3}\right)$ is given below.

Proposition 4.6. If the action:

$$
J=\iint L d^{3} x d t=\iint L \sqrt{g} d^{3} q d t \equiv \iint \bar{L} d^{3} q d t,
$$

is invariant under the infinitesimal Lie transformation:

$$
z^{\prime}=z^{s}+\epsilon V^{s}, \quad q^{\prime \alpha}=q^{\alpha}+\epsilon V^{q^{\alpha}}, \quad(0 \leqslant \alpha \leqslant 3, \quad 1 \leqslant s \leqslant N),
$$

and under the divergence transformation:

$$
\bar{L}^{\prime}=\bar{L}+\epsilon \nabla \cdot \overline{\boldsymbol{\Lambda}}, \quad \overline{\boldsymbol{\Lambda}}=\sqrt{g} \boldsymbol{\Lambda}, \quad \bar{L}=\sqrt{g} L,
$$

then the Euler Lagrange equations (4.54) admit the conservation law:

$$
\frac{1}{\sqrt{g}} \frac{\partial}{\partial q^{\alpha}}\left\{\sqrt{g}\left(V^{q^{\alpha}} L+\hat{V}^{z^{j}} L_{j}^{\alpha}+\Lambda^{\alpha}\right)\right\}=0 .
$$

Proof. The proof follows the usual derivation of Noether's theorem (e.g. Bluman and Kumei (1989); Webb et al. (2005)). Note that $q^{\alpha}=\left(t, q^{1}, q^{2}, q^{3}\right)$. 
The completes our discussion of the multi-symplectic system (4.49).

\section{Multi-Symplectic MHD}

In this section we develop multi-symplectic approaches to the MHD equations. In Section 5.1, we develop a multi-symplectic form of the MHD equations using the magnetic induction $\mathbf{B}$ as the basic variable describing the magnetic field and by using Clebsch variables. This leads formally to 15 variable set of equations in the state vector $z=$ $\left(\mathbf{u}^{T}, \rho, S, \mu, \mathbf{B}^{T}, \boldsymbol{\Gamma}^{T}, \lambda, \beta, \phi\right)^{T}$ in which $\mu, \boldsymbol{\Gamma}, \lambda, \beta, \phi$ are Clebsch variables and $\rho, \mathbf{u}, \mathbf{B}$ and $S$ are physical variables. In Section 5.2 we develop a similar multi-symplectic formulation of the MHD equations, except that the magnetic vector potential $\mathbf{A}$, in which $\alpha=\mathbf{A} \cdot d \mathbf{x}$ is Lie dragged with the background flow is used to describe the magnetic field and $\mathbf{B}=\nabla \times \mathbf{A}$ is the magnetic field induction. In this latter formulation $\mathbf{Z}=\left(\mathbf{u}^{T}, \rho, S, \mu\right.$, $\left.\mathbf{A}^{T}, \gamma^{T}, \lambda, \beta, \phi\right)^{T}$ is the state vector of the system and $\mu, \gamma, \lambda, \beta$, and $\phi$ are Clebsch potentials. We show that there is a direct map between the state vector $\mathbf{z}$ of Section 5.1 and the state vector $\mathbf{Z}$ of Section 5.2.

\subsection{Advected Magnetic Flux Case}

In the Clebsch variables approach, the fluid velocity is given by the expression:

$$
\rho \mathbf{u}=\rho \nabla \phi-\beta \nabla S-\lambda \nabla \mu-(\nabla \times \mathbf{\Gamma}) \times \mathbf{B}-\boldsymbol{\Gamma}(\nabla \cdot \mathbf{B}),
$$

In the standard Clebsch variable formulation (Section 3.1), in which $t$ is the evolution variable, the canonical coordinates are the physical variables $\left(\rho, S, \mu, \mathbf{B}^{\mathbf{T}}\right)$ and the Lagrange multipliers $\left(\phi, \beta, \lambda, \boldsymbol{\Gamma}^{T}\right)$ are the corresponding canonical momenta (the role of the canonical momenta and coordinates can be interchanged, simply by changing the sign of the Hamiltonian). In the multi-symplectic formulation both space and time can be thought of as evolution variables.

In the multi-symplectic approach used in the present analysis, the Clebsch variable expansion for the fluid velocity $\mathbf{u}$ in (5.1) is re-written in the form:

$$
\beta \nabla S+\lambda \nabla \mu+\boldsymbol{\Gamma}(\nabla \cdot \mathbf{B})+\mathbf{B} \cdot \nabla \boldsymbol{\Gamma}-\mathbf{B} \cdot(\nabla \boldsymbol{\Gamma})^{T}-\rho \nabla \phi=-\rho \mathbf{u} \equiv-\frac{\delta \ell}{\delta \mathbf{u}},
$$

where

$$
\ell=\int_{V}\left(\frac{1}{2} \rho|\mathbf{u}|^{2}-\varepsilon(\rho, S)-\frac{B^{2}}{2 \mu_{0}}\right) d^{3} x
$$

is the MHD Lagrangian without constraints.

Proposition 5.1. The evolution equations (3.17)-(3.21) and the Clebsch variable equation (5.2) for $-\delta \ell / \delta \mathbf{u}$ can be written in the multi-symplectic form:

$$
\left(\mathbf{K}^{0} \frac{\partial}{\partial t}+\mathbf{K}^{1} \frac{\partial}{\partial x}+\mathbf{K}^{2} \frac{\partial}{\partial y}+\mathbf{K}^{3} \frac{\partial}{\partial z}\right) \mathbf{z}=\frac{\delta \mathcal{H}}{\delta \mathbf{z}},
$$

where $\mathrm{A}$ is a $15 \times 15$ matrix differential operator. In (5.4)

$$
\mathbf{z}=\left(\mathbf{u}^{T}, \rho, S, \mu, \mathbf{B}^{T}, \boldsymbol{\Gamma}^{T}, \lambda, \beta, \phi\right)^{T},
$$

is a 15-dimensional state vector for the system and the $\mathrm{K}^{\alpha}(\alpha=0,1,2,3)$ are skewsymmetric $15 \times 15$ matrices, and

$$
\mathcal{H}=-\ell \equiv-\int_{V}\left(\frac{1}{2} \rho|\mathbf{u}|^{2}-\varepsilon(\rho, S)-\frac{B^{2}}{2 \mu_{0}}\right) d^{3} x=\int_{V} H(\mathbf{z}) d^{3} x
$$


is the multi-symplectic Hamiltonian functional for the system. The functional or variational derivative $\delta \mathcal{H} / \delta z^{s}=\partial H / \partial z^{s}$ in the present case. The skew-symmetric matrices $\mathrm{K}^{\alpha}$ satisfy equations of the form:

$$
\mathrm{K}_{i j}^{\alpha} d z^{i} \wedge d z^{j}=d \omega^{\alpha} \quad \text { where } \omega^{\alpha}=L_{j}^{\alpha} d z^{j}
$$

are symplectic one-forms. For the MHD system, the one-forms $\omega^{\alpha}$ are given by (up the exterior derivative of a scalar function):

$$
\begin{aligned}
\omega^{0} & =\phi d \rho+\beta d S+\lambda d \mu+\boldsymbol{\Gamma} \cdot d \mathbf{B}, \\
\omega^{i} & =[\mathbf{u}(\beta d S+\lambda d \mu+\phi d \rho)+\rho \phi d \mathbf{u}+(\boldsymbol{\Gamma} \cdot \mathbf{B}) d \mathbf{u}-\mathbf{B}(\boldsymbol{\Gamma} \cdot d \mathbf{u})+\mathbf{u}(\boldsymbol{\Gamma} \cdot d \mathbf{B})]^{i}, \\
& \equiv[\mathbf{u}(\beta d S+\lambda d \mu-\rho d \phi)+d(\rho \phi \mathbf{u})+(\boldsymbol{\Gamma} \cdot \mathbf{u}) d \mathbf{B}-(\boldsymbol{\Gamma} \times d \mathbf{E})]^{i}
\end{aligned}
$$

where $1 \leqslant i \leqslant 3$ and

$$
\mathbf{E}=-\mathbf{u} \times \mathbf{B}
$$

is the electric field in ideal MHD. The adjoint $\mathrm{A}^{\dagger}$ of the matrix differential operator $\mathrm{A}$ satisfies the equation:

$$
\boldsymbol{\psi}^{T} \cdot \mathrm{A} \mathbf{z}=\frac{\partial}{\partial x^{\alpha}}\left(\boldsymbol{\psi}^{T} \cdot \mathrm{K}^{\alpha} \mathbf{z}\right)+\mathbf{z}^{T} \cdot \mathrm{A}^{\dagger} \boldsymbol{\psi}
$$

where

$$
\mathrm{A}^{\dagger} \boldsymbol{\psi}=\frac{\partial}{\partial x^{\alpha}}\left(\mathrm{K}^{\alpha} \boldsymbol{\psi}\right)
$$

Note that $\langle\boldsymbol{\psi}, \mathrm{A} \mathbf{z}\rangle=\left\langle\mathbf{z}, \mathrm{A}^{\dagger} \boldsymbol{\psi}\right\rangle$, where $\langle$,$\rangle is the usual inner product.$

Proof.To derive (5.4)-(5.9) first note that the Clebsch variable equation (5.2) for $\rho \mathbf{u}$ and the evolution equations (3.17)-(3.21) can be written in the form:

$$
\begin{aligned}
& \beta \nabla S+\lambda \nabla \mu+\boldsymbol{\Gamma}(\nabla \cdot \mathbf{B})+\mathbf{B} \cdot \nabla \boldsymbol{\Gamma}-\mathbf{B} \cdot(\nabla \boldsymbol{\Gamma})^{T}-\rho \nabla \phi=H_{\mathbf{u}}, \\
& -D_{t} \phi=H_{\rho}, \quad-\beta \nabla \cdot \mathbf{u}-D_{t} \beta=H_{S}, \quad-\lambda \nabla \cdot \mathbf{u}-D_{t} \lambda=H_{\mu}, \\
& -\boldsymbol{\Gamma} \cdot(\nabla \mathbf{u})^{T}-D_{t} \boldsymbol{\Gamma}=H_{\mathbf{B}}, \quad \mathbf{B}(\nabla \cdot \mathbf{u})-\mathbf{B} \cdot \nabla \mathbf{u}+D_{t} \mathbf{B}=H_{\boldsymbol{\Gamma}}, \\
& D_{t} \mu=H_{\lambda}, \quad D_{t} S=H_{\beta}, \quad \rho \nabla \cdot \mathbf{u}+D_{t} \rho=H_{\phi},
\end{aligned}
$$

where $D_{t}=\partial_{t}+\mathbf{u} \cdot \nabla$ is the Lagrangian time derivative and the multi-symplectic Hamiltonian is given by (5.6). In (5.14) we use the notation $H_{\psi} \equiv \partial H / \partial \psi$.

To obtain the matrices $\mathrm{K}^{\alpha}$ in (5.4) write (5.14) in the matrix form:

$$
\mathrm{A} \mathbf{z}=H_{\mathbf{z}} \quad \text { where } \quad \mathrm{A}=\mathrm{K}^{\alpha} \frac{\partial}{\partial x^{\alpha}},
$$

and $\left(x^{0}, x^{1}, x^{2}, x^{3}\right) \equiv(t, x, y, z)$. Note that the equations involving $H_{\mathbf{u}}, H_{\mathbf{B}}$ and $H_{\boldsymbol{\Gamma}}$ each consist of three equations, but the other equations involving $H_{\rho}, H_{S}, H_{\mu}, H_{\lambda}, H_{\beta}$ and $H_{\phi}$ are single equations. The matrix differential operator A in (5.15) has the form:

$$
\mathrm{A}=\left(\begin{array}{ccccccccc}
\mathrm{O}_{3 \times 3} & 0 & \beta \nabla & \lambda \nabla & \boldsymbol{\Gamma} \nabla \cdot & \mathbf{V}_{\mathbf{B}} & 0 & 0 & -\rho \nabla \\
\mathrm{O}_{1 \times 3} & 0 & 0 & 0 & \mathrm{O}_{1 \times 3} & \mathrm{O}_{1 \times 3} & 0 & 0 & -D_{t} \\
-\beta \nabla \cdot & 0 & 0 & 0 & \mathrm{O}_{1 \times 3} & \mathrm{O}_{1 \times 3} & 0 & -D_{t} & 0 \\
-\lambda \nabla \cdot & 0 & 0 & 0 & \mathrm{O}_{1 \times 3} & \mathrm{O}_{1 \times 3} & -D_{t} & 0 & 0 \\
-\boldsymbol{\Gamma} \cdot(\nabla \circ)^{T} & 0 & 0 & 0 & \mathrm{O}_{3 \times 3} & -\mathrm{I}_{3 \times 3} D_{t} & 0 & 0 & 0 \\
-V_{\mathbf{B}}^{\dagger} & 0 & 0 & 0 & \mathrm{O}_{3 \times 3} D_{t} & \mathrm{O}_{3 \times 3} & 0 & 0 & 0 \\
\mathrm{O}_{1 \times 3} & 0 & 0 & D_{t} & \mathrm{O}_{1 \times 3} & \mathrm{O}_{1 \times 3} & 0 & 0 & 0 \\
\mathrm{O}_{1 \times 3} & 0 & D_{t} & 0 & \mathrm{O}_{1 \times 3} & \mathrm{O}_{1 \times 3} & 0 & 0 & 0 \\
\rho \nabla \cdot & D_{t} & 0 & 0 & \mathrm{O}_{1 \times 3} & \mathrm{O}_{1 \times 3} & 0 & 0 & 0
\end{array}\right)
$$


where

$$
V_{\mathbf{B}}=\mathbf{B} \cdot \nabla \circ-\mathbf{B} \cdot(\nabla \circ)^{T}, \quad V_{\mathbf{B}}^{\dagger}=\mathbf{B} \cdot(\nabla \circ)^{T}-\mathbf{B} \nabla \cdot \circ .
$$

In (5.16) $\mathrm{O}_{3 \times 3}$ is the zero $3 \times 3$ matrix, $\mathrm{I}_{3 \times 3}$ is the unit $3 \times 3$ unit matrix and $\mathrm{O}_{1 \times 3}$ is the $1 \times 3$ zero matrix. The operator:

$$
D_{t}=\frac{\partial}{\partial t}+\mathbf{u} \cdot \nabla
$$

is the Lagrangian time derivative following the flow. Note that $V_{\mathbf{B}}^{\dagger}$ is the adjoint of the operator $V_{\mathbf{B}}$ with respect to the usual inner product $\langle f, g\rangle=\int f g d^{3} x$ for real functions (further discussion of $V_{\mathbf{B}}^{\dagger}$ is given in Appendix B).

Using (5.16) the skew symmetric matrices $\mathrm{K}_{i j}^{\alpha}$ have the form:

$$
\mathrm{K}_{i j}^{\alpha}=\mathrm{k}_{[i, j]}^{\alpha}=\mathrm{k}_{i j}^{\alpha}-\mathrm{k}_{j i}^{\alpha} \text {. }
$$

In particular:

$$
\mathrm{k}_{i j}^{0}=\delta_{15}^{i} \delta_{4}^{j}+\delta_{14}^{i} \delta_{5}^{j}+\delta_{13}^{i} \delta_{6}^{j}+\delta_{10}^{i} \delta_{7}^{j}+\delta_{11}^{i} \delta_{8}^{j}+\delta_{12}^{i} \delta_{9}^{j}
$$

Similarly:

$$
\begin{aligned}
\mathrm{k}_{i j}^{1}= & \Gamma^{x} \delta_{1}^{i} \delta_{7}^{j}+\Gamma^{y} \delta_{2}^{i} \delta_{7}^{j}+\Gamma^{z} \delta_{3}^{i} \delta_{7}^{j} \\
& +B^{x}\left(\delta_{2}^{i} \delta_{11}^{j}+\delta_{3}^{i} \delta_{12}^{j}\right)+B^{y} \delta_{11}^{i} \delta_{1}^{j}+B^{z} \delta_{12}^{i} \delta_{1}^{j}+u^{x}\left(\delta_{10}^{i} \delta_{7}^{j}+\delta_{11}^{i} \delta_{8}^{j}+\delta_{12}^{i} \delta_{9}^{j}\right) \\
& +\left\{u^{x}\left(\delta_{14}^{i} \delta_{5}^{j}+\delta_{13}^{i} \delta_{6}^{j}+\delta_{15}^{i} \delta_{4}^{j}\right)+\beta \delta_{1}^{i} \delta_{5}^{j}+\lambda \delta_{1}^{i} \delta_{6}^{j}-\rho \delta_{1}^{i} \delta_{15}^{j}\right\}, \\
\mathrm{k}_{i j}^{2}= & \Gamma^{x} \delta_{1}^{i} \delta_{8}^{j}+\Gamma^{y} \delta_{2}^{i} \delta_{8}^{j}+\Gamma^{z} \delta_{3}^{i} \delta_{8}^{j} \\
& +B^{x} \delta_{10}^{i} \delta_{2}^{j}+B^{y}\left(\delta_{1}^{i} \delta_{10}^{j}+\delta_{3}^{i} \delta_{12}^{j}\right)+B^{z} \delta_{12}^{i} \delta_{2}^{j}+u^{y}\left(\delta_{10}^{i} \delta_{7}^{j}+\delta_{11}^{i} \delta_{8}^{j}+\delta_{12}^{i} \delta_{9}^{j}\right) \\
& +\left\{u^{y}\left(\delta_{13}^{i} \delta_{6}^{j}+\delta_{14}^{i} \delta_{5}^{j}+\delta_{15}^{i} \delta_{4}^{j}\right)+\beta \delta_{2}^{i} \delta_{5}^{j}+\lambda \delta_{2}^{i} \delta_{6}^{j}-\rho \delta_{2}^{i} \delta_{15}^{j}\right\}, \\
k_{i j}^{3}= & \Gamma^{x} \delta_{1}^{i} \delta_{9}^{j}+\Gamma^{y} \delta_{2}^{i} \delta_{9}^{j}+\Gamma^{z} \delta_{3}^{i} \delta_{9}^{j} \\
& +B^{x} \delta_{10}^{i} \delta_{3}^{j}+B^{y} \delta_{11}^{i} \delta_{3}^{j}+B^{z}\left(\delta_{1}^{i} \delta_{10}^{j}+\delta_{2}^{i} \delta_{11}^{j}\right)+u^{z}\left(\delta_{10}^{i} \delta_{7}^{j}+\delta_{11}^{i} \delta_{8}^{j}+\delta_{12}^{i} \delta_{9}^{j}\right) \\
& +\left\{u^{z}\left(\delta_{13}^{i} \delta_{6}^{j}+\delta_{14}^{i} \delta_{5}^{j}+\delta_{15}^{i} \delta_{4}^{j}\right)+\beta \delta_{3}^{i} \delta_{5}^{j}+\lambda \delta_{3}^{i} \delta_{6}^{j}-\rho \delta_{3}^{i} \delta_{15}^{j}\right\} .
\end{aligned}
$$
i.e.

The one-form solutions for $\omega^{\alpha}=L_{j}^{\alpha} d z^{j}$ in (5.8)-(5.9) are related to the $\mathrm{K}_{j k}^{\alpha}$ by (4.14),

$$
\mathrm{K}_{j k}^{\alpha}=\frac{\partial L_{k}^{\alpha}}{\partial z^{j}}-\frac{\partial L_{j}^{\alpha}}{\partial z^{k}}
$$

Note that the solution of (5.24) for the $L_{j}^{\alpha}$ are not unique because $\omega^{\alpha}=L_{j}^{\alpha} d z^{j}+d \Phi(\mathbf{z})^{\alpha}$ will also give the same $\mathrm{K}_{j k}^{\alpha}$.

As an example we find $\omega^{0}=L_{j}^{0} d z^{j}$ is given by:

$$
\begin{aligned}
\omega^{0} & =\left(z^{15} d z^{4}+z^{14} d z^{5}+z^{13} d z^{6}\right)+\left\{z^{10} d z^{7}+z^{11} d z^{8}+z^{12} d z^{9}\right\} \\
& \equiv \phi d \rho+\beta d S+\lambda d \mu+\mathbf{\Gamma} \cdot d \mathbf{B} .
\end{aligned}
$$

Similarly, we obtain (5.9) for $\omega^{i}$.

\subsubsection{Exterior differential forms approach}

Although the above derivation of the MHD multi-symplectic structure is straightforward, there is some ambiguity in the one forms $\omega^{0}$ and $\omega^{i}$ in (5.8)-(5.9) since one can 
always add a perfect differential to these forms. A more elegant way to derive the above results of Proposition 5.1 is to use differential forms to deduce the skew symmetric matrices $\mathrm{K}^{\alpha}$ and the one forms $\omega^{\alpha}(\alpha=0,1,2,3)$ describing the system. This approach is described below. From (3.14) the MHD Lagrangian may be written in the form:

$$
L=\frac{1}{2} \rho u^{2}-\varepsilon(\rho, S)-\frac{B^{2}}{2 \mu}+L_{z^{s}}^{\alpha} \frac{\partial z^{s}}{\partial x^{\alpha}},
$$

where

$$
\begin{aligned}
L_{z^{s}}^{\alpha} \frac{\partial z^{s}}{\partial x^{\alpha}}= & \phi\left(\frac{\partial \rho}{\partial t}+\nabla \cdot(\rho \mathbf{u})\right)+\beta\left(\frac{\partial S}{\partial t}+\mathbf{u} \cdot \nabla S\right)+\lambda\left(\frac{\partial \mu}{\partial t}+\mathbf{u} \cdot \nabla \mu\right) \\
& +\boldsymbol{\Gamma} \cdot\left(\frac{\partial \mathbf{B}}{\partial t}-\nabla \times(\mathbf{u} \times \mathbf{B})+\mathbf{u}(\nabla \cdot \mathbf{B})\right) .
\end{aligned}
$$

In particular:

$$
L_{z^{s}}^{0} \frac{\partial z^{s}}{\partial x^{0}}=\phi \rho_{t}+\beta S_{t}+\lambda \mu_{t}+\boldsymbol{\Gamma} \cdot \mathbf{B}_{t}
$$

and hence

$$
\begin{aligned}
\omega^{0} & =L_{\rho}^{0} d \rho+L_{S}^{0} d S+L_{\mu}^{0} d \mu+L_{\mathbf{B}}^{0} \cdot d \mathbf{B}, \\
& \equiv \phi d \rho+\beta d S+\lambda d \mu+\boldsymbol{\Gamma} \cdot d \mathbf{B},
\end{aligned}
$$

(note (5.29) define the non-zero $\left.L_{z^{s}}^{0}\right)$. The result (5.29) for $\omega^{0}$ is the same as (5.8). Taking the exterior derivative of (5.29) gives:

$$
d \omega^{0}=d \phi \wedge d \rho+d \beta \wedge d S+d \lambda \wedge d \mu+d \Gamma_{s} \wedge d B^{s} \equiv \frac{1}{2} K_{z^{s}, z^{p}}^{0} d z^{s} \wedge d z^{p} .
$$

Hence

$$
\mathrm{K}_{\phi, \rho}^{0}=\mathrm{K}_{\beta, S}^{0}=\mathrm{K}_{\lambda, \mu}^{0}=\mathrm{K}_{\Gamma_{s}, B^{s}}^{0}=1 \quad(s=1,2,3) .
$$

Thus we obtain the skew symmetric matrix $\mathrm{K}_{i j}^{0}$ given by (5.19) and (5.20).

A similar calculation gives:

$$
\begin{aligned}
L_{z^{s}}^{k} \frac{\partial z^{s}}{\partial x^{k}}= & \phi\left(\rho \nabla_{k} u^{k}+u^{k} \nabla_{k} \rho\right)+\beta\left(u^{k} \nabla_{k} S\right)+\lambda u^{k} \nabla_{k} \mu \\
& +\Gamma_{s}\left(u^{k} \nabla_{k} B^{s}+B^{s} \nabla_{k} u^{k}-B^{k} \nabla_{k} u^{s}\right),
\end{aligned}
$$

from which we read off:

$$
\begin{aligned}
& L_{\rho}^{k}=\phi u^{k}, \quad L_{u^{i}}^{k}=(\rho \phi+\boldsymbol{\Gamma} \cdot \mathbf{B}) \delta_{i}^{k}-\Gamma_{i} B^{k}, \\
& L_{S}^{k}=\beta u^{k}, \quad L_{\mu}^{k}=\lambda u^{k}, \quad L_{B^{i}}^{k}=\Gamma_{i} u^{k},
\end{aligned}
$$

Using (5.33) we obtain:

$$
\omega^{k}=L_{z^{s}}^{k} d z^{s}=\{\mathbf{u}[\phi d \rho+\beta d S+\lambda d \mu]+\rho \phi d \mathbf{u}+(\boldsymbol{\Gamma} \cdot \mathbf{B}) d \mathbf{u}-\mathbf{B}(\boldsymbol{\Gamma} \cdot d \mathbf{u})+\mathbf{u}(\boldsymbol{\Gamma} \cdot d \mathbf{B})\}^{k},
$$

which is the result (5.9) for $\omega^{k}$. Taking the exterior derivative of (5.34) gives:

$$
\begin{aligned}
d \omega^{k}= & d u^{k} \wedge\left(\beta d S+\lambda d \mu-\rho d \phi-B^{s} d \Gamma_{s}\right) \\
& +u^{k}\left(d \phi \wedge d \rho+d \beta \wedge d S+d \lambda \wedge d \mu+d \Gamma_{s} \wedge d B^{s}\right) \\
& -\Gamma_{s} d B^{k} \wedge d u^{s}-B^{k} d \Gamma_{s} \wedge d u^{s} .
\end{aligned}
$$


From (5.35) we obtain:

$$
\begin{aligned}
& \mathrm{K}_{u^{k}, S}^{k}=\beta, \quad \mathrm{K}_{u^{k}, \mu}^{k}=\lambda, \quad \mathrm{K}_{u^{k}, \phi}^{k}=-\rho, \\
& \mathrm{K}_{\Gamma_{s}, B^{s}}^{k}=\mathrm{K}_{\phi, \rho}^{k}=\mathrm{K}_{\beta, S}^{k}=\mathrm{K}_{\lambda, \mu}^{k}=u^{k}, \quad \mathrm{~K}_{u^{s}, B^{k}}^{k}=\Gamma_{s}, \\
& \mathrm{~K}_{u^{k}, \Gamma_{s}}^{k}=-B^{s}, \quad \mathrm{~K}_{u^{s}, \Gamma_{s}}^{k}=B^{k} \quad(k \neq s) .
\end{aligned}
$$

By using the state vector $\mathbf{z}=\left(\mathbf{u}^{T}, \rho, S, \mu, \mathbf{B}^{T}, \boldsymbol{\Gamma}^{T}, \lambda, \beta, \phi\right)^{T}$ and (5.36) gives the results (5.21)-(5.23) for the $\mathrm{K}_{i j}^{k}(k=1,2,3)$.

\subsubsection{Multi-Symplectic Conservation Laws}

Proposition 5.2. Using the results (5.8) and (5.10) for the one-forms $\omega^{0}$ and $\omega^{k}(k=$ $1,2,3)$, the multi-symplectic conservation law (4.21) for $\beta=0$ reduces to:

$$
\frac{\partial D}{\partial t}+\nabla \cdot \mathbf{F}=0
$$

where

$$
\begin{aligned}
& D=\left(\frac{1}{2} \rho|\mathbf{u}|^{2}+\varepsilon(\rho, S)+\frac{B^{2}}{2 \mu_{0}}\right)-\nabla \cdot(\mathbf{E} \times \boldsymbol{\Gamma}+\rho \phi \mathbf{u}), \\
& \mathbf{F}=\mathbf{u}\left(\frac{1}{2} \rho|\mathbf{u}|^{2}+\varepsilon(\rho, S)+p\right)+\frac{\mathbf{E} \times \mathbf{B}}{\mu_{0}}+\frac{\partial}{\partial t}(\mathbf{E} \times \boldsymbol{\Gamma}+\rho \phi \mathbf{u})-\nabla \times[(\boldsymbol{\Gamma} \cdot \mathbf{u}) \mathbf{E}] .
\end{aligned}
$$

Because of null divergence terms in (5.38), the conservation law (5.37) reduces to the MHD energy conservation equation:

$$
\frac{\partial}{\partial t}\left(\frac{1}{2} \rho|\mathbf{u}|^{2}+\varepsilon(\rho, S)+\frac{B^{2}}{2 \mu_{0}}\right)+\nabla \cdot\left(\mathbf{u}\left(\frac{1}{2} \rho|\mathbf{u}|^{2}+\varepsilon(\rho, S)+p\right)+\frac{\mathbf{E} \times \mathbf{B}}{\mu_{0}}\right)=0 .
$$

Similarly, the multi-symplectic conservation law (4.21) for $\beta=k$ gives a conservation law of the form (5.37) but with

$$
\begin{aligned}
D \equiv D^{k}= & -\rho u^{k}+\nabla_{k}(\rho \phi+\boldsymbol{\Gamma} \cdot \mathbf{B})-\nabla \cdot\left(\Gamma^{k} \mathbf{B}\right) \\
F^{i} \equiv F^{i k}= & -\left\{\rho u^{i} u^{k}+\left(p+\frac{B^{2}}{2 \mu_{0}}\right) \delta^{i k}-\frac{B^{i} B^{k}}{\mu_{0}}\right\} \\
& +\left[-\frac{\partial}{\partial t}(\rho \phi+\boldsymbol{\Gamma} \cdot \mathbf{B}) \delta^{i k}+\frac{\partial}{\partial t}\left(\Gamma^{k} B^{i}\right)\right] \\
& +\nabla \times\left(\Gamma^{k} \mathbf{E}\right)^{i}+\nabla_{k}\left[\boldsymbol{\Gamma} \cdot \mathbf{B} u^{i}\right]-\nabla \cdot[\boldsymbol{\Gamma} \cdot \mathbf{B u}] \delta^{i k} .
\end{aligned}
$$

The conservation law (5.37) reduces to:

$$
-\left\{\frac{\partial}{\partial t}(\rho \mathbf{u})+\nabla \cdot\left[\rho \mathbf{u} \otimes \mathbf{u}+\left(p+\frac{B^{2}}{2 \mu_{0}}\right) \mathbf{I}-\frac{\mathbf{B} \otimes \mathbf{B}}{\mu_{0}}\right]\right\}^{k}=0
$$

i.e., the conservation law reduces to the MHD momentum conservation equation in the $x^{k}$ - direction.

Proof.The multi-symplectic Hamiltonian density $H$, and the 1 -forms $\omega^{\alpha}=\Gamma_{j}^{\alpha} d z^{j}$ from (5.8) and (5.9) give:

$$
\begin{aligned}
& L_{j}^{0} z_{, \alpha}^{j}=\phi \rho_{, \alpha}+\beta S_{, \alpha}+\lambda \mu_{, \alpha}+\Gamma_{s} B_{, \alpha}^{s}, \\
& L_{j}^{i} z_{, \alpha}^{j}=u^{i}\left[\beta S_{, \alpha}+\lambda \mu_{, \alpha}-\rho \phi_{, \alpha}\right]+\boldsymbol{\Gamma} \cdot \mathbf{u} B_{, \alpha}^{i}+\left(\rho \phi u^{i}\right)_{, \alpha}-\epsilon_{i j k} \Gamma^{j} E_{, \alpha}^{k} \quad(\alpha=0,1,2,3) .
\end{aligned}
$$


Using (5.42) we obtain:

$$
\begin{aligned}
L & =L_{j}^{\alpha} z_{, \alpha}^{j}-H \equiv p-\frac{B^{2}}{2 \mu_{0}}+\frac{\partial}{\partial t}(\rho \phi)+\nabla \cdot(\rho \phi \mathbf{u}), \\
H & =-\left(\frac{1}{2} \rho|\mathbf{u}|^{2}-\varepsilon(\rho, S)-\frac{B^{2}}{2 \mu_{0}}\right) .
\end{aligned}
$$

Using the results (5.42)-(5.43) in the symplectic conservation law (4.21) for $\beta=0$ and $\beta=k$ gives the energy and momentum conservation laws (5.39) and (5.41).

Proposition 5.3. The symplecticity or structural conservation laws (4.22) for MHD and gas dynamics can be written in the form:

$$
D_{\alpha}\left(F_{a b}^{\alpha}\right)=0, \quad a<b
$$

where

$$
F_{a b}^{\alpha}=\mathbf{z}_{, a}^{T} \mathrm{~K}^{\alpha} \mathbf{z}_{, b} .
$$

$F_{a b}^{\alpha}$ can be calculated by noting that:

$$
d \omega^{\alpha}=F_{a b}^{\alpha} d x^{a} \otimes d x^{b} \quad \text { where } \quad a<b .
$$

Using (5.29) and (5.34) for $\omega^{0}$ and $\omega^{i}(i=1,2,3)$ and calculating $d \omega^{0}$ and $d \omega^{i}$ gives the formulae:

$$
\begin{aligned}
F_{a b}^{0}= & \frac{\partial(\phi, \rho)}{\partial\left(x^{a}, x^{b}\right)}+\frac{\partial(\beta, S)}{\partial\left(x^{a}, x^{b}\right)}+\frac{\partial(\lambda, \mu)}{\partial\left(x^{a}, x^{b}\right)}+\frac{\partial\left(\Gamma_{s}, B^{s}\right)}{\partial\left(x^{a}, x^{b}\right)} \\
F_{a b}^{i}= & \frac{\partial\left(\phi u^{i}, \rho\right)}{\partial\left(x^{a}, x^{b}\right)}+\frac{\partial\left(\beta u^{i}, S\right)}{\partial\left(x^{a}, x^{b}\right)}+\frac{\partial\left(\lambda u^{i}, \mu\right)}{\partial\left(x^{a}, x^{b}\right)} \\
& +\frac{\partial\left(\rho \phi, u^{i}\right)}{\partial\left(x^{a}, x^{b}\right)}+\frac{\partial\left(u^{i} \Gamma_{s}, B^{s}\right)}{\partial\left(x^{a}, x^{b}\right)}-\frac{\partial\left(B^{i} \Gamma_{s}, u^{s}\right)}{\partial\left(x^{a}, x^{b}\right)} .
\end{aligned}
$$

Proof.We give the derivation of (5.47). Using (5.30) we obtain:

$$
\begin{aligned}
d \omega^{0} & =\left(\frac{\partial \phi}{\partial x^{a}} \frac{\partial \rho}{\partial x^{b}}+\frac{\partial \beta}{\partial x^{a}} \frac{\partial S}{\partial x^{b}}+\frac{\partial \lambda}{\partial x^{a}} \frac{\partial \mu}{\partial x^{b}}+\frac{\partial \Gamma_{s}}{\partial x^{a}} \frac{\partial B^{s}}{\partial x^{b}}\right) d x^{a} \wedge d x^{b} \\
& =\left\{\frac{\partial(\phi, \rho)}{\partial\left(x^{a}, x^{b}\right)}+\frac{\partial(\beta, S)}{\partial\left(x^{a}, x^{b}\right)}+\frac{\partial(\lambda, \mu)}{\partial\left(x^{a}, x^{b}\right)}+\frac{\partial\left(\Gamma_{s}, B^{s}\right)}{\partial\left(x^{a}, x^{b}\right)}\right\} d x^{a} \otimes d x^{b} .
\end{aligned}
$$

Using (5.49) we obtain the formula (5.47) for $F_{a b}^{0}$.

A similar analysis gives:

$$
d \omega^{i}=F_{a b}^{i} d x^{a} \otimes d x^{b}, \quad(i=1,2,3),
$$

where $F_{a b}^{i}$ is given by (5.48). This completes the proof.

\subsection{Advected $\mathbf{A} \cdot d \mathbf{x}$ formulation}

In Section 3.2, we discussed the MHD variational equations that result when the condition that the one-form $\alpha=\mathbf{A} \cdot d \mathbf{x}$ is advected with the background flow is used instead of Faraday's equation which is equivalent to the condition that the magnetic flux 2-form $\beta=\mathbf{B} \cdot d \mathbf{S}$ is Lie dragged with the flow, for the case where $\nabla \cdot \mathbf{B}=0$ and $\mathbf{B}=\nabla \times \mathbf{A}$ (e.g., Tur and Yanovsky (1993), Webb et al. (2014a)). Note that $\beta=d \alpha=\mathbf{B} \cdot d \mathbf{S}$ in the above case. In addition

$$
H=\int_{V} \alpha \wedge \beta=\int_{V}(\mathbf{A} \cdot d \mathbf{x}) \wedge(\mathbf{B} \cdot d \mathbf{S})=\int_{V} \mathbf{A} \cdot \mathbf{B} d^{3} x,
$$


is the Hopf invariant or magnetic helicity (note if there is not a global $\mathbf{A}$ with $\mathbf{B}=\nabla \times \mathbf{A}$, then the magnetic field has a non-trivial topology, e.g. Moffatt (1969), Arnold and Khesin (1998)).

Proposition 5.4. Choose the gauge of $\mathbf{A}$ such that $\alpha=\mathbf{A} \cdot d \mathbf{x}$, is advected with the background flow. The Clebsch variational equations (3.34)-(3.36) imply:

$$
\begin{aligned}
& \beta \nabla S+\lambda \nabla \mu+\gamma \cdot(\nabla \mathbf{A})^{T}-\gamma \cdot \nabla \mathbf{A}-\mathbf{A}(\nabla \cdot \gamma)-\rho \nabla \phi=\mathcal{H}_{\mathbf{u}} \equiv-\rho \mathbf{u}, \\
& -D_{t} \phi=\mathcal{H}_{\rho} \equiv-\left(\frac{1}{2}|\mathbf{u}|^{2}-h\right), \quad-\beta \nabla \cdot \mathbf{u}-D_{t}(\beta)=\mathcal{H}_{S} \equiv \rho T, \\
& -\lambda \nabla \cdot \mathbf{u}-D_{t} \lambda=\mathcal{H}_{\mu} \equiv 0, \quad-\gamma \nabla \cdot \mathbf{u}+(\boldsymbol{\gamma} \cdot \nabla) \mathbf{u}-D_{t} \boldsymbol{\gamma}=\mathcal{H}_{\mathbf{A}} \equiv \mathbf{J}, \\
& \mathbf{A} \cdot(\nabla \mathbf{u})^{T}+D_{t} \mathbf{A}=\mathcal{H}_{\gamma} \equiv 0, \quad D_{t} \mu=\mathcal{H}_{\lambda}=0, \\
& D_{t} S=\mathcal{H}_{\beta} \equiv 0, \quad \rho \nabla \cdot \mathbf{u}+D_{t} \rho=\mathcal{H}_{\phi} \equiv 0,
\end{aligned}
$$

where $\mathbf{J}=\nabla \times \mathbf{B} / \mu_{0}$ is the MHD currrent,

$$
\mathcal{H}=-\ell=-\int_{V}\left(\frac{1}{2} \rho|\mathbf{u}|^{2}-\varepsilon(\rho, S)-\frac{|\nabla \times \mathbf{A}|^{2}}{2 \mu_{0}}\right) d^{3} x \equiv \int_{V} H(\mathbf{Z}) d^{3} x,
$$

is the multi-symplectic Hamiltonian and

$$
\mathbf{Z}=\left(\mathbf{u}^{T}, \rho, S, \mu, \mathbf{A}^{T}, \gamma^{T}, \lambda, \beta, \phi\right)^{T},
$$

is the 15-dimensional state vector for the system. The transformations:

$$
\Gamma^{*}=-\mathbf{A}, \quad \mathbf{B}^{*}=\gamma,
$$

formally maps the left handsides of the advected $\mathbf{A}$ variational equations (3.34)-(3.36) (i.e. (5.52)) onto the Clebsch variational equations (5.14) associated with the advection of the magnetic flux $\mathbf{B} \cdot d \mathbf{S}$, where to obtain (5.14) the replacements $\boldsymbol{\Gamma}^{*} \rightarrow \boldsymbol{\Gamma}$ and $\mathbf{B}^{*} \rightarrow \mathbf{B}$ are made.

Proof.To verify that the map (5.55) maps the Clebsch equations (5.52) associated with the Lie dragging of $\mathbf{A} \cdot d \mathbf{x}$ onto the Clebsch equations (5.14), it suffices to consider only the equations related to the evolution of $\mathbf{A}$ and $\gamma$ and of $\mathbf{B}$ and $\boldsymbol{\Gamma}$. The first equation in (5.52) under the map (5.55) becomes:

$$
\beta \nabla S+\lambda \nabla \mu-\mathbf{B}^{*} \cdot\left(\nabla \boldsymbol{\Gamma}^{*}\right)^{T}+\left(\mathbf{B}^{*} \cdot \nabla\right) \boldsymbol{\Gamma}^{*}+\boldsymbol{\Gamma}^{*}\left(\nabla \cdot \mathbf{B}^{*}\right)-\rho \nabla \phi=\mathcal{H}_{\mathbf{u}},
$$

which is the first equation in (5.14), but with $\mathbf{B} \rightarrow \mathbf{B}^{*}$ and $\boldsymbol{\Gamma} \rightarrow \boldsymbol{\Gamma}^{*}$.

Similarly, the $5^{\text {th }}$ equation in (5.52) becomes:

$$
-\left\{\mathbf{B}^{*}(\nabla \cdot \mathbf{u})-\left(\mathbf{B}^{*} \cdot \nabla\right) \mathbf{u}+D_{t} \mathbf{B}^{*}\right\}=-\mathcal{H}_{\mathbf{\Gamma}^{*}},
$$

which is equivalent to the sixth equation in (5.14).

The sixth equation in (5.52) becomes:

$$
-\boldsymbol{\Gamma}^{*} \cdot(\nabla \mathbf{u})^{T}-D_{t} \boldsymbol{\Gamma}^{*}=\mathcal{H}_{\mathbf{B}^{*}},
$$

which is the $5^{t h}$ equation in (5.14), but with $\boldsymbol{\Gamma} \rightarrow \boldsymbol{\Gamma}^{*}$ and $\mathbf{B} \rightarrow \mathbf{B}^{*}$. This completes the proof.

RemarkThe Hamiltonian for the system (5.52) is given by (5.53). Under the transformations (5.55) the Hamiltonian functional $\mathcal{H}$ becomes:

$$
\mathcal{H}=-\int_{V}\left(\frac{1}{2} \rho|\mathbf{u}|^{2}-\varepsilon(\rho, S)-\frac{\left|\nabla \times \boldsymbol{\Gamma}^{*}\right|^{2}}{2 \mu_{0}}\right) d^{3} x .
$$


Proposition 5.5. The one forms describing the multi-symplectic MHD system, using the advected $\mathbf{A} \cdot d \mathbf{x}$ formalism are:

$$
\begin{aligned}
& \omega^{0}=\phi d \rho+\beta d S+\lambda d \mu+\gamma_{s} d A^{s}, \\
& \omega^{k}=u^{k}(\beta d S+\lambda d \mu+\phi d \rho)+\phi \rho d u^{k}+\gamma_{k} A^{s} d u^{s}+u^{k} \gamma_{s} d A^{s}, \quad k=1,2,3,
\end{aligned}
$$

The forms (5.60) and (5.61) can then be used to determine the skew symmetric matrices $\mathrm{K}^{\alpha}$, for $\alpha=0,1,2,3$.

Proof.Using the action principle (3.33) we can write:

$$
L=\frac{1}{2} \rho u^{2}-\varepsilon(\rho, S)-\frac{|\nabla \times \mathbf{A}|^{2}}{2 \mu}+L_{Z^{s}}^{\alpha} \frac{\partial Z^{s}}{\partial x^{\alpha}},
$$

where

$$
\begin{aligned}
L_{Z^{s}}^{\alpha} \frac{\partial Z^{s}}{\partial x^{\alpha}}= & \phi\left[\rho_{t}+\nabla(\rho \mathbf{u})\right]+\beta\left(S_{t}+\mathbf{u} \cdot \nabla S\right)+\lambda\left(\mu_{t}+\mathbf{u} \cdot \nabla \mu\right) \\
& +\gamma \cdot\left[\mathbf{A}_{t}-\mathbf{u} \times(\nabla \times \mathbf{A})+\nabla(\mathbf{u} \cdot \mathbf{A})\right] .
\end{aligned}
$$

Following the approach in (5.26) et seq., we identify:

$$
\begin{aligned}
\omega^{0} & =L_{\rho}^{0} d \rho+L_{S}^{0} d S+L_{\mu}^{0} d \mu+L_{\mathbf{A}}^{0} \cdot d \mathbf{A}, \\
& \equiv \phi d \rho+\beta d S+\lambda d \mu+\gamma_{s} d A^{s},
\end{aligned}
$$

and hence:

$$
L_{\rho}^{0}=\phi, \quad L_{S}^{0}=\beta, \quad L_{\mu}^{0}=\lambda, \quad L_{A^{s}}^{0}=\gamma_{s} .
$$

Taking the exterior derivative of (5.64) gives:

$$
d \omega^{0}=d \phi \wedge d \rho+d \beta \wedge d S+d \lambda \wedge d \mu+d \gamma_{s} \wedge d A^{s},
$$

which implies:

$$
\mathrm{K}_{\phi, \rho}^{0}=\mathrm{K}_{\beta, S}^{0}=\mathrm{K}_{\lambda, \mu}^{0}=\mathrm{K}_{\gamma_{s}, A^{s}}^{0}=1 .
$$

Similarly using (5.63) we obtain:

$$
\omega^{k}=L_{Z^{s}}^{k} d Z^{s}=u^{k}(\beta d S+\lambda d \mu+\phi d \rho)+\phi \rho d u^{k}+\gamma_{k} A^{s} d u^{s}+u^{k} \gamma_{s} d A^{s},
$$

and hence:

$$
\begin{aligned}
& L_{S}^{k}=\beta u^{k}, \quad L_{\mu}^{k}=\lambda u^{k}, \quad L_{\rho}^{k}=\phi u^{k}, \quad L_{u^{k}}^{k}=\phi \rho+\gamma_{k} A^{k}, \\
& L_{u^{s}}^{k}=\gamma_{k} A^{s}, \quad L_{A^{s}}^{k}=u^{k} \gamma_{s} .
\end{aligned}
$$

Taking the exterior derivative of (5.68) gives:

$$
\begin{aligned}
d \omega^{k}= & d u^{k}[\beta d S+\lambda d \mu+\phi d \rho]+u^{k}[d \beta \wedge d S+d \lambda \wedge d \mu+d \phi \wedge d \rho]+(\phi d \rho+\rho d \phi) \wedge d u^{k} \\
& +\left(d \gamma_{k} A^{s}+\gamma_{k} d A^{s}\right) \wedge d u^{s}+\left(d u^{k} \gamma_{s}+u^{k} d \gamma_{s}\right) \wedge d A^{s},
\end{aligned}
$$

which can be used to determine the $\mathrm{K}_{Z^{\mu}, Z^{\nu}}^{k}$, i.e.

$$
\begin{aligned}
& \mathrm{K}_{u^{k}, S}^{k}=\beta, \quad \mathrm{K}_{u^{k}, \mu}^{k}=\lambda, \\
& \mathrm{K}_{\beta, S}^{k}=\mathrm{K}_{\lambda, \mu}^{k}=\mathrm{K}_{\phi, \rho}^{k}=u^{k}, \quad \mathrm{~K}_{\phi, u^{k}}^{k}=\rho, \\
& \mathrm{K}_{A^{s}, u^{s}}^{k}=\gamma_{k}, \quad \mathrm{~K}_{u^{k}, A^{s}}^{k}=\gamma_{s}, \quad(k \neq s), \\
& \mathrm{K}_{\gamma_{k}, u^{s}}^{k}=A^{s}, \quad \mathrm{~K}_{\gamma_{k}, A^{s}}^{k}=u^{k} .
\end{aligned}
$$


Proposition 5.6. The structural conservation laws for the advected $\mathbf{A} \cdot d \mathbf{x}$ version of the MHD equations is given by (5.44) in which:

$$
\begin{aligned}
F_{a b}^{0}= & \frac{\partial(\phi, \rho)}{\partial\left(x^{a}, x^{b}\right)}+\frac{\partial(\beta, S)}{\partial\left(x^{a}, x^{b}\right)}+\frac{\partial(\lambda, \mu)}{\partial\left(x^{a}, x^{b}\right)}+\frac{\partial\left(\gamma_{s}, A^{s}\right)}{\partial\left(x^{a}, x^{b}\right)} \\
F_{a b}^{i}= & \frac{\partial\left(\phi u^{i}, \rho\right)}{\partial\left(x^{a}, x^{b}\right)}+\frac{\partial\left(\beta u^{i}, S\right)}{\partial\left(x^{a}, x^{b}\right)}+\frac{\partial\left(\lambda u^{i}, \mu\right)}{\partial\left(x^{a}, x^{b}\right)} \\
& +\frac{\partial\left(\rho \phi, u^{i}\right)}{\partial\left(x^{a}, x^{b}\right)}+\frac{\partial\left(\gamma_{i} A^{s}, u^{s}\right)}{\partial\left(x^{a}, x^{b}\right)}+\frac{\partial\left(\gamma_{s} A^{i}, A^{s}\right)}{\partial\left(x^{a}, x^{b}\right)}
\end{aligned}
$$

$(i=1,2,3$ and $a, b=0,1,2,3)$ are the conserved densities and fluxes respectively where $a<b$.

Proof.The proof is the same as in Proposition 5.3, except that $\omega^{0}$ and $\omega^{i}$ are now given by $(5.60)$ and (5.61).

\section{Summary and Concluding Remarks}

In this paper we developed multi-symplectic equations for ideal MHD. A key ingredient was the use of Clebsch variable variational principles in which the constraint equations (mass continuity, entropy advection, Lin constraint, Faraday's equation, or its analogue for the magnetic vector potential $\mathbf{A}$ ), are ensured by using Lagrange multipliers. The connection between Clebsch variables and the momentum map in ideal fluid systems with constraints was used (Section 3.1). The Lin constraint can be viewed as defining a Lagrangian variable which is advected with the flow (more generally it is useful to include 3 Lagrangian labels, to include different possible initial data, that are not included in the usual Clebsch variable description (see e.g. Cotter et al. (2007)).

Section 2 introduces the MHD equations. In Section 2 we also discuss one dimensional gas dynamics as an example of a multi-symplectic system. The example illustrates, that both time and space can be thought of as evolution variables. The example uses Clebsch variables to describe the gas dynamic equations. The multi-symplectic formulation involves two skew symmetric matrices associated with the time and space evolution. The gas dynamic equations are obtained by finding the stationary point conditions for the action, including the mass continuity equation and entropy advection equation constraints by using Lagrange multipliers. One forms (i.e. differential forms) are constructed from the constraint equations which lead to the multi-symplectic form of the 1D gas dynamic equations. The theory implies that there are in general extra conservation laws that arise from using an expanded phase space involving the Clebsch variables. In 1D gas dynamics the symplecticity conservation law involves the space derivative of the energy conservation law, and the time derivative of the momentum conservation law. In this case and in more general cases (Sections 4 and 5), the extra symplecticity conservation laws imposes constraints that ensure that the equations involve the same effective number of dependent variables as the original Eulerian formulation of the fluid equations. The conserved densities and fluxes are written in terms of $2 \times 2$ Jacobians of the dependent variables with respect to two of the independent space-time variables.

Two different formulations were investigated. In the first formulation, the constraint that the magnetic flux $\beta=\mathbf{B} \cdot d \mathbf{S}$ is Lie dragged (i.e. conserved moving with the flow), 
leads to Faraday's equation in the form:

$$
\frac{\partial \mathbf{B}}{\partial t}-\nabla \times(\mathbf{u} \times \mathbf{B})+\mathbf{u}(\nabla \cdot \mathbf{B})=0,
$$

(for mathematical reasons it is useful to consider the case $\nabla \cdot \mathbf{B} \neq 0$ as well as the physical case $\nabla \cdot \mathbf{B}=0)$. An alternative method to account for Faraday's equation, is to require that the gauge of the magnetic vector potential $\mathbf{A}$ is chosen so that the 1 -form $\alpha=\mathbf{A} \cdot d \mathbf{x}$ is Lie dragged by the flow, i.e. A satisfies the evolution equation:

$$
\frac{\partial \mathbf{A}}{\partial t}-\mathbf{u} \times(\nabla \times \mathbf{A})+\nabla(\mathbf{u} \cdot \mathbf{A})=0 .
$$

For the case where $\mathbf{A}$ satisfies $(6.2), \mathbf{A} \cdot \mathbf{B} / \rho$ is a scalar invariant advected by the flow, i.e.

$$
\frac{d}{d t}\left(\frac{\mathbf{A} \cdot \mathbf{B}}{\rho}\right)=0
$$

where $d / d t=\partial / \partial t+\mathbf{u} \cdot \nabla$ is the Lagrangian time derivative following the flow and $\mathbf{B}=$ $\nabla \times \mathbf{A}$.

Following the approach of Cotter et al. (2007), we showed that the Clebsch variable evolution equations and the Clebsch representation for the the mass flux (momentum density) $\rho \mathbf{u}$ could be written in the multi-symplectic form:

$$
\mathrm{K}_{i j}^{\alpha} \frac{\partial z^{j}}{\partial x^{\alpha}}=\frac{\delta \mathcal{H}}{\delta z^{i}},
$$

where $x^{\alpha}(\alpha=0,1,2,3)$ denote the space-time coordinates $(t, x, y, z)$ and

$\mathbf{z}=\left(\mathbf{u}^{T}, \rho, S, \mu, \mathbf{B}^{T}, \boldsymbol{\Gamma}^{T}, \lambda, \beta, \phi\right)^{T}$ is a 15 -dimensional state vector describing the system. The covariant form of the multi-symplectic system (6.4) for non-Cartesian spatial coordinates was discussed in Section 4 (see also Bridges et al. (2010)). The multi-symplectic Hamiltonian in (6.4), given by

$$
\mathcal{H}=-\ell=-\int_{V}\left(\frac{1}{2} \rho|\mathbf{u}|^{2}-\varepsilon(\rho, S)-\frac{B^{2}}{2 \mu_{0}}\right) d^{3} x,
$$

is the negative of the MHD Lagrangian functional without constraints. The skew symmetric matrices $\mathrm{K}_{i j}^{\alpha}(1 \leqslant i, j \leqslant 15)$ are related to one-forms $\omega^{\alpha}$, by the equations:

$$
\kappa^{\alpha}=\frac{1}{2} \mathrm{~K}_{i j}^{\alpha}(\mathbf{z}) d z^{i} \wedge d z^{j}=d \omega^{\alpha} \quad \text { and } \quad \omega^{\alpha}=L_{j}^{\alpha} d z^{j},
$$

where the equation $\kappa_{; \alpha}^{\alpha}=0$ is the symplecticity conservation law.

Section 4 discusses multi-symplectic systems, based in part on the work of Hydon (2005). This included a discussion of skew symmetric operators and matrices and Poisson brackets in Hamiltonian systems in which the time is the evolution variable. The conservation of the phase space element following the Hamiltonian flow and its generalization for multi-symplectic systems (i.e. the symplecticity conservation law) were derived. Noether's theorem for multi-symplectic systems was discussed, and the form of the equations for generalized non-Cartesian space coordinates were studied (see Bridges et al. (2010) for a study of multi-symplectic systems and the variational bi-complex using the total exterior algebra tangent bundle (TEA)). Proposition 4.3 shows that multi-symplectic systems can be written as a Cartan-Poincaré form equation using an $N+2$-form where $N$ is the number of independent space variables (see also Marsden and Shkoller (1999)), which involves the one-forms $\omega^{\alpha}$ and their exterior derivatives $d \omega^{\alpha}(0 \leqslant \alpha \leqslant N)$ and the multi-symplectic Hamiltonian $H$. The Cartan-Poincaré form can be related to Cartan's geometric formulation of partial differential equations (e.g. Harrison and Estabrook 
(1971)). Multi-symplectic systems for generalized (Cartesian and non-Cartesian) space coordinates were derived from the variational principle.

In Section 5, we demonstrated that the multi-symplectic MHD equations obtained using the magnetic vector potential A satisfying (6.2) are related to the multi-symplectic MHD equations using $\mathbf{B}$ and Faraday's law (6.1) by the transformations (5.55), i.e.

$$
\Gamma^{*}=-\mathbf{A} \text { and } \mathbf{B}^{*}=\gamma .
$$

In (6.7) $\psi^{*}$ is the image of $\psi$ under the map. $\mathbf{A}$ is the magnetic vector potential and $\gamma$ is the corresponding Lagrange multiplier in the advected $\mathbf{A}$ variational principle (3.33). $\mathbf{B}=\nabla \times \mathbf{A}$ and $\boldsymbol{\Gamma}$ is the Lagrange multiplier for Faraday's equation in the variational principle (3.13). Under the map (6.7), $\gamma \rightarrow \mathbf{B}^{*} \equiv \mathbf{B}$ and $\mathbf{A} \rightarrow-\boldsymbol{\Gamma}^{*} \equiv-\boldsymbol{\Gamma}$. In Section 5 we also obtained the 6 symplecticity conservation laws that occur when there are 4 independent (space-time) variables. These conservation laws are obtained from setting combinations of the derivatives of the momentum and energy conservation equations equal to zero, which ensures conservation of phase space. These conservation laws have densities $D$ and fluxes $F$ that consist of a sum of $2 \times 2$ Jacobians of the dependent variables with respect to the space-time coordinates. One can also derive conservation laws using the multisymplectic versions of Noether's theorems. In particular, it is possible to derive the generalized non-local cross helicity conservation law for MHD and the generalized non-local helicity conservation law for ideal fluids, that apply for non-barotropic equations of state for the gas. These conservation laws were derived in Webb et al. (2014b) (paper II) using Noether's theorem, fluid relabelling symmetries and gauge symmetries for the Lagrangian. They depend on the nonlocal Clebsch variables. For barotropic gases, these conservation laws reduce to the usual local cross helicity conservation law for MHD, and the helicity conservation law for ideal fluids. However, because the Eulerian fluid velocity variation (Lie symmetry generator) for relabelling symmetries is zero, this implies a constraint on the Clebsch variable symmetry generators (see e.g. Calkin (1963)). Non-local conservation laws in partial differential equation systems can arise from Lie potential symmetries of the cover system of equations, consisting of the the original system augmented by the differential equations for the potentials of the original system (e.g. Bluman et al. (2010), Sjöberg and Mahomed, (2004), Webb and Zank (2009)). This is worth further investigation.

The Clebsch variable approach to MHD and fluid equations used in the present paper is not necessarily the only way that multi-symplectic systems of equations can be derived. Bridges (2006) in a study of elliptic partial differential equations (pdes) using the total exterior algebra bundle (TEA) makes the interesting observation that the symplectic matrices can sometimes have more obvious symmetry properties when higher order matrices are used to describe the system (e.g. the quaternion algebra is revealed when using $4 \times 4$ matrices to describe the $2 \mathrm{D}$ Klein Gordon equation, which is not obvious when $3 \times 3$ matrices are used to describe the multi-symplectic structure).

\section{Aknowledgements}

GMW acknowledges stimulating discussions of MHD conservation laws and multisymplectic systems with Darryl Holm. We acknowledge discussions of the noncanonical MHD Poisson bracket and multi-symplectic MHD with Phillip Morrison. GPZ was supported in part by NASA grants NN05GG83G and NSF grant nos. ATM-03-17509 and ATM-04-28880. JFMcK acknowledges support by the NRF of South Africa. 


\section{Appendix A}

In this appendix we derive the momentum and energy conservation equations (2.24) and (2.25) for one dimensional gas dynamics using the symplecticity pullback conservation laws (4.21). We also derive the structural or simplecticity conservation law (2.26) using (4.22).

The energy and momentum conservation laws (2.24) and (2.25) follow from (4.21), i.e.,

$$
D_{\alpha}\left(L_{j}^{\alpha}(z) z_{, \nu}^{j}-L \delta_{\nu}^{\alpha}\right)=0
$$

where the Lagrange density $L$ is given by (2.18) and the one-forms $\omega^{\alpha}=L_{z^{s}}^{\alpha} d z^{s}$ are given by (2.22) and $\alpha=0,1$ and $\nu=0,1$ for $1 \mathrm{D}$ gas dynamics and $\mathbf{z}=(u, \rho, S, \beta, \phi)^{T}$ are the dependent variables, $x^{0}=t$ and $x^{1}=x$. The fluid velocity $u$ is given by the Clebsch form (2.10), i.e.

$$
u=\frac{\partial \phi}{\partial x}-\frac{\beta}{\rho} \frac{\partial S}{\partial x} .
$$

For $\nu=0$ (A 1) gives the energy conservation equation:

$$
\frac{\partial D_{0}}{\partial t}+\frac{\partial F_{0}}{\partial x}=0
$$

where

$$
D_{0}=L_{z^{j}}^{0} z_{, 0}^{j}-L, \quad F_{0}=L_{z^{j}}^{1} z_{, 0}^{j},
$$

are the conserved density $D_{0}$ and flux $F_{0}$. The Lagrange density $L$ is given by (2.18)$(2.19)$, i.e.:

$$
L=\frac{1}{2} \rho u^{2}-\varepsilon(\rho, S)+\phi\left[\frac{\partial \rho}{\partial t}+\frac{\partial}{\partial x}(\rho u)\right]+\beta\left(\frac{\partial S}{\partial t}+u \frac{\partial S}{\partial x}\right) .
$$

From (A 4) we obtain:

$$
D_{0}=\phi \rho_{t}+\beta S_{t}-L, \quad F_{0}=u \phi \rho_{t}+\beta u S_{t}+\phi \rho u_{t} .
$$

Using $L$ from (A 5) and using the Clebsch expansion (A 2) for $u$ in (A 6) we obtain:

$$
\begin{aligned}
& D_{0}=\frac{1}{2} \rho u^{2}+\varepsilon(\rho, S)-D_{x}(\rho u \phi), \\
& F_{0}=D_{t}(\rho u \phi)+\rho u\left(\frac{1}{2} u^{2}+h\right) .
\end{aligned}
$$

Substitution of $D_{0}$ and $F_{0}$ from (A 7) in (A 3) gives the energy conservation law (2.24).

The momentum conservation law from (A 1$)$ has the form: $D_{t}\left(D_{1}\right)+D_{x}\left(F_{1}\right)=0$ where

$$
D_{1}=L_{z^{j}}^{0} z_{, 1}^{j}, \quad F_{1}=L_{z^{j}}^{1} z_{, 1}^{j}-L .
$$

We find:

$$
\begin{aligned}
D_{1} & =\phi \rho_{x}+\beta S_{x} \equiv D_{x}(\rho \phi)-\rho u, \\
F_{1} & =u \phi \rho_{x}+u \beta S_{x}+\phi \rho u_{x}-L \\
& =-\left(\rho u^{2}+p\right)-D_{t}(\rho \phi)-\beta\left(S_{t}+u S_{x}\right) .
\end{aligned}
$$

From (A 8)-(A 9) we obtain:

$$
\frac{\partial D_{1}}{\partial t}+\frac{\partial F_{1}}{\partial x}=-\left[\frac{\partial}{\partial t}(\rho u)+\frac{\partial}{\partial x}\left(\rho u^{2}+p\right)\right]=0,
$$

which is the momentum conservation equation (2.25). 
In general, the symplecticity conservation laws are given by (4.22), i.e.

$$
D_{\alpha}\left(F_{\nu \gamma}^{\alpha}\right)=0, \text { where } \nu<\gamma,
$$

and

$$
F_{\nu \gamma}^{\alpha}=\mathrm{K}_{i j}^{\alpha} z_{, \nu}^{i} z_{, \gamma}^{j} .
$$

For the case of 1D gas dynamics, there is only one structural conservation law, namely:

$$
D_{t}\left(F_{01}^{0}\right)+D_{x}\left(F_{01}^{1}\right)=0 .
$$

Using (A 12) we find:

$$
\begin{aligned}
F_{01}^{0} & =z_{, 0}^{i} \mathbf{K}_{i j}^{0} z_{, 1}^{j} \equiv\left[\mathbf{z}_{t}\right]^{T} \mathbf{K}^{0} \mathbf{z}_{x} \\
& =\phi_{t} \rho_{x}+\beta_{t} S_{x}-S_{t} \beta_{x}-\phi_{x} \rho_{t} \\
& =\frac{\partial(\phi, \rho)}{\partial(t, x)}+\frac{\partial(\beta, S)}{\partial(t, x)} .
\end{aligned}
$$

Similarly we find:

$$
\begin{aligned}
F_{01}^{1} & =z_{, 0}^{i} \mathrm{~K}_{i j}^{1} z_{, 1}^{j} \equiv\left[\mathbf{z}_{t}\right]^{T} \mathrm{~K}^{1} \mathbf{z}_{x} \\
& =\left(\rho \phi_{t}-\beta S_{t}\right) u_{x}+u \phi_{t} \rho_{x}+\frac{\partial}{\partial t}(\beta \rho) S_{x}-u S_{t} \beta_{x}-\phi_{x} \frac{\partial}{\partial t}(\rho u) \\
& =\rho \frac{\partial(\phi, u)}{\partial(t, x)}+u \frac{\partial(\phi, \rho)}{\partial(t, x)}+\beta \frac{\partial(u, S)}{\partial(t, x)}+u \frac{\partial(\beta, S)}{\partial(t, x)},
\end{aligned}
$$

Using (A 14) and (A 15) in (A 13) gives the symplecticity conservation law (2.26).

\section{Appendix B}

In this appendix we discuss the notation

$$
V_{\mathbf{B}}=\mathbf{B} \cdot \nabla \circ-\mathbf{B} \cdot(\nabla \circ)^{T}, \quad V_{\mathbf{B}}^{\dagger}=\mathbf{B} \cdot(\nabla \circ)^{T}-\mathbf{B}(\nabla \cdot \circ),
$$

used in (5.17). Consider the integral:

$$
\begin{aligned}
\int_{R} V_{\mathbf{B}}(\mathbf{W}) d^{3} x & =\int_{R}\left[\mathbf{B} \cdot \nabla \circ-\mathbf{B} \cdot(\nabla \circ)^{T}\right] \mathbf{W} d^{3} x \\
& =\int_{R}\left(B^{s} \frac{\partial W^{i}}{\partial x^{s}}-B^{s} \nabla_{i} W^{s}\right) \mathbf{e}_{i} d^{3} x \\
& =\int_{R}\left\{\nabla \cdot(\mathbf{B} \otimes \mathbf{W})-\nabla(\mathbf{B} \cdot \mathbf{W})-\mathbf{W}(\nabla \cdot \mathbf{B})+\mathbf{W} \cdot(\nabla \mathbf{B})^{T}\right\} d^{3} x \\
& =\int_{R}\left\{\mathbf{W} \cdot(\nabla \circ)^{T}-\mathbf{W} \nabla \cdot \circ\right\} \mathbf{B} d^{3} x=\int_{R} V_{\mathbf{W}}^{\dagger}(\mathbf{B}) d^{3} x,
\end{aligned}
$$

where

$$
V_{\mathbf{W}}^{\dagger}=\mathbf{W} \cdot(\nabla \circ)^{T}-\mathbf{W}(\nabla \cdot \circ)
$$

and the $\left\{\mathbf{e}_{i}\right\}$ are unit base vectors along the $x, y$ and $z$ axes. In the derivation of (B 2) we have used Gauss' divergence theorem to obtain:

$$
\begin{aligned}
\int_{R} \nabla \cdot(\mathbf{B} \otimes \mathbf{W}) d^{3} x & =\int_{\partial R}(\mathbf{B} \cdot \mathbf{n}) \mathbf{W} d \mathbf{S}, \\
\int_{R} \nabla(\mathbf{B} \cdot \mathbf{W}) d^{3} x & =\int_{\partial R}(\mathbf{B} \cdot \mathbf{W}) \mathbf{n} d S,
\end{aligned}
$$


where $\mathbf{n}$ is the outward normal to the region $R$, and we assume that the surface integrals (B 4) vanish. The formula $V_{\mathbf{B}}^{\dagger}$ in (B 1) now follows by using the replacement $\mathbf{W} \rightarrow \mathbf{B}$ in (B 3).

\section{REFERENCES}

Akhmetiev, P., and Ruzmaikin, A. 1995, A fourth order topological invariant of magnetic or vortex lines, J. Geom. Phys. 15, 95-101.

Anderson, I. M. 1989, The Variational Bicomplex, book manuscript; Utah State University (1989). http : //www.math.usu.edu/ $f g_{m} p /$ Publications/VB/vb.pdf

Anderson, I. M. 1992, Introduction to the variational Bi-complex. in Mathematical aspects of contemporary field theory, Contemp. Math., 132, 51-73.

Arnold, V. I. and Khesin, B. A. 1998, Topological Methods in Hydrodynamics, Springer, New York.

Berger, M. A. 1990, Third -order link integrals, J. Phys. A: Math. Gen., 23, 2787-2793.

Berger, M. A. and Field, G. B., 1984, The toplological properties of magnetic helicity, J. Fluid. Mech., 147, 133-148.

Bluman, G. W. and Kumei, S. 1989, Symmetries and Differential Equations, (New York: Springer).

Bluman, G. W., Cheviakov, A. F. and Anco, S. 2010, Applications of Symmetry Methods to Partial Differential Equations, Springer: New York.

Bridges, T. J. 1992, Spatial Hamiltonian structure, energy flux and the water-wave problem. Proc. Roy. Soc. London,439, 297-315.

Bridges, T.J., 1997a, Multi-symplectic structures and wave propagation, Math. Proc. Camb. Philos. Soc., 121, 147-190.

Bridges, T. J., 1997b, A geometric formulation of the conservation of wave action and its implications for signature and classification of instabilities, Proc. Roy. Soc. A, 453, 1365-1395 (1997b).

Bridges, T. J. Hydon, P. E. and Reich, S. 2005, Vorticity and symplecticity in Lagrangian fluid dynamics, J. Phys. A: Math. Gen. 38 1403-1418 (2005)

Bridges, T. J. and Reich, S., 2006, Numerical methods for Hamiltonian PDEs, J. Phys. A, Math. Gen., 39, 5287-5320.

Bridges, T. J. 2006, Canonical multi-symplectic structure on the total exterior algebra bundle, Proc. Roy. Soc. London, A, 462, 1531-1551.

Bridges, T. J., Hydon, P. E. and Lawson, J.K. 2010, Multi-symplectic structures and the variational bi-complex, Math. Proc. Cambridge Phil. Soc., issue 1, (Jan. 2010), pp 159-178.

Brio, M., Zakharian, A. R. and Webb, G. M. 2010, Numerical Time-Dependent Partial Differential Equations for Scientists and Engineers, Mathematics in science and Engineering, vol.123, Elsevier Press, (Ed. C. K. Chui) 2010, first edition, pp. 199-204.

Calkin, M. G. 1963, An action principle for magnetohydrodynamics, Canad.J. Phys., 41, 22412251.

Chandre, C., de Guillebon, L., Back, A., Tassi, E. and Morrison, P. J. 2013, On the use of projectors for Hamiltonian systems and their relationship with Dirac brackets, J. Phys. A, Math. and theoret., 46, 125203 (14pp), doi:10.10.1088/1751-8133/46/12/125203.

Cotter, C. J., Holm, D. D., and Hydon, P. E., 2007, Multi-symplectic formulation of fluid dynamics using the inverse map, Proc. Roy. Soc. Lond. A, 463, 2617-2687 (2007).

Finn, J. H. and Antonsen, T. M. 1985, Magnetic helicity: what is it and what is it good for?, Comments on Plasma Phys. and Contr. Fusion, 9(3), 111.

Finn, J. M. and Antonsen, T. M. 1988, Magnetic helicity injection for configurations with field errors, Phys. Fluids, 31 (10), 3012-3017.

Gordin, V. A. and Petviashvili, V. I., 1987, The gauge of vector potential and Lyapunov stable MHD equilibrium, Soviet J. Plasma Phys., 13, No. 7,pp. 509-511 (English).

Harrison, B. K. and Estabrook, F. B., 1971, Geometric approach to invariance groups and solution of partial differential systems, J. Math. Phys., 12, 653-66.

Holm, D. D. and Kupershmidt, B. A. 1983a, Poisson brackets and Clebsch representations for magnetohydrodynamics, multi-fluid plasmas and elasticity, Physica D, 6D, 347-363. 
Holm, D. D. and Kupershmidt, B. A. 1983b, Noncanonical Hamiltonian formulation of ideal magnetohydrodynamics, Physica D, 7D, 330-333.

Holm, D.D., Marsden, J.E. and Ratiu, T.S. 1998, The Euler-Lagrange equations and semiproducts with application to continuum theories, Adv. Math., 137, 1-81.

Hydon, P. E., 2005, Multi-symplectic conservation laws for differential and differential-difference equations, Proc. Roy. Soc. A, 461, 1627-1637 (2005).

Hydon, P. E. and Mansfield, E. L. 2011, Extensions of Noether's second theorem: from continuous to discrete systems, Proc. Roy. Soc. A, 467, pp. 3206-3221, doi:10.1098/rspa.2011.0158.

Marsden J. E. and Ratiu T.S. 1994, Introduction to Mechanics and Symmetry, New York,: Springer Verlag.

Marsden, J. E. and Shkoller, S. 1999, Multi-symplectic geometry, covariant Hamiltonians and Water Waves, Math. Proc. Camb. Phil. Soc., 125, 553-575.

Moffatt, H. K. 1969, The degree of knottedness of tangled vortex lines, J. Fluid. Mech., 35, 117.

Morrison, P. J. 1982, Poisson brackets for fluids and plasmas, in Mathematical Methods in Hydrodynamics and Integrability of dynamical Systems, AIP Proc. Conf., 88, ed M. Tabor and Y. M. Treve, pp 13-46.

Morrison, P.J. and Greene, J.M. 1980, Noncanonical Hamiltonian density formulation of hydrodynamics and ideal magnetohydrodynamics, Phys. Rev. Lett., 45, 790-794.

Morrison, P.J. and Greene, J.M. 1982, Noncanonical Hamiltonian density formulation of hydrodynamics and ideal magnetohydrodynamics, (Errata), Phys. Rev. Lett., 48, 569.

Morrison, P. J. 1998, Hamiltonian description of the ideal fluid, Reviews of Modern Physics, 70, Issue 2, April 1998, pp.467-521, 10.1103/RevModPhys.70.467

Newcomb, W. A. 1962, Lagrangian and Hamiltonian methods in magnetohydrodynamics, Nucl. Fusion Suppl., Part 2, 451-463.

Padhye, N. S. and Morrison, P. J. 1996a, Fluid relabeling symmetry, Phys. Lett. A, 219, 287-292.

Padhye, N. S. and Morrison, P. J. 1996b, Relabeling symmetries in hydrodynamics and magnetohydrodynamics, Plasma Phys. Reports, 22, 869-877.

Powell, K. G., Roe, P.L., Linde, T.J., Gombosi, T. I., and De Zeeuw, D. 1999, A solution adaptive upwind scheme for ideal magnetohydrodynamics, J. Comput. Phys. 154, 284-309.

Reich, S. 2000, Multi-symplectic Runge-Kutta Collocation methods for Hamiltonian wave equations, J. Comp. Phys., 57, 473.

Ruzmaikin, A. and Akhmetiev, P. 1994, Topological invariants of magnetic fields and the effect of reconnections, Phys. Plasmas, 1, No. 2, 331-338.

Sjöberg, A. and Mahomed, F.M. 2004, Non-local symmetries and conservation laws for onedimensional gas dynamics equations, Appl. Math. Comput., 150, 379-397.

Tur, A. V. and Yanovsky, V. V. 1993, Invariants in dissipationless hydrodynamic media, J. Fluid Mech., 248, Cambridge Univ. Press, p67-106.

Webb, G. M., Zank, G.P., Kaghashvili, E. Kh and Ratkiewicz, R.E., 2005, Magnetohydrodynamic waves in non-uniform flows II: stress energy tensors, conservation laws and Lie symmetries, J. Plasma Phys., 71, 811-857, doi: 10.1017/S00223778050003740.

Webb, G. M.; McKenzie, J. F.; Mace, R. L.; Ko, C. M.; Zank, G. P. 2007, Dual variational principles for nonlinear traveling waves in multifluid plasmas, Phys. of Plasmas, 4, Issue 8, pp. 082318-082318-17, doi:10.1063/1.2757154

Webb, G. M., Ko, C. M., Mace, R.L., McKenzie, J.F. and Zank, G.P. 2008, Integrable, oblique travelling waves in charge neutral, two-fluid plasmas, Nonl. Proc. Geophys., 15, 179-208.

Webb, G. M. and Zank, G. P. 2009, Scaling symmetries, conservation laws and action principles in one-dimensional gas dynamics, J. Phys. A., Math. Theor., 42, 475205 (23pp).

Webb, G.M., Pogorelov, N.V. and Zank, G.P. 2010, MHD simple waves and the divergence wave, Solar Wind, 12, St. Malo, France, AIP Proc. Conf., 1216, pp.300-303. doi:10.1063/1.3396300.

Webb, G. M., Dasgupta, B., McKenzie, J. F., Hu, Q., and Zank, G.P. 2014a: Local and nonlocal advected invariants and helicities in magnetohydrodynamics and gas dynamics I: Lie dragging approach, J. Phys. A., Math. and Theoret., 47, (2014) 095501 (33pp). doi:10.1088/1751-8113/49/9/095501, preprint available at http://arxiv.org/abs/1307.1105.

Webb, G. M., Dasgupta, B., McKenzie, J. F., Hu, Q., and Zank, G.P. 2014b: Local and nonlocal advected invariants and helicities in magnetohydrodynamics and gas dynamics II: Noether's 
theorems and Casimirs, J. Phys. A., Math. and Theoret., 47 (2014) 095502 (31pp), doi:10.1088/1751-8113/47/9/095502, preprint available at http://arxiv.org/abs/1307.1038.

Webb, G. M., Hu, Q., McKenzie, J. F., Dasgupta, B. and Zank, G.P. 2014c, Advected invariants in MHD and gas dynamics, 12th Ann. Internat. Astrophys. Conf., in Outstanding Problems in Heliophysics: from Coronal Heating to the Edge of the Heliosphere, eds. G.P. Zank and Q. Hu, Astronomical Society of the Pacific Conf. Series, 484, 229-234.

Webb, G. M., Burrows, R. H., Ao, X., and Zank, G.P. 2014d, Ion acoustic travelling waves, J. Plasma Phys., 80, part 2, pp. 147-171, doi:10.1017/S0022377813001013, preprint at http://arxiv.org/abs/1312.6406.

Webb, G. M. and Mace, R.L. 2014, Noether's Theorems and fluid relabelling symmetries in magnetohydrodynamics and gas dynamics, J. Phys. A, Math. and Theoret., article JPHYSA101-057, submitted March 10, 2014, available at http://arxiv.org/abs/1403.3133

Woltjer, L., On hydromagnetic equilibria, Proc. Nat. Acad. of Sciences, 44, No. 9, 833-841.

Zakharov, V. E. and Kuznetsov, E.A. 1997, Hamiltonian formalism for nonlinear waves, PhysicsUspekhi, 40, (11), 1087-1116. 